\title{
A local character based method for solving linear systems of radiation diffusion problems ${ }^{\text {th }}$
}

\author{
Shuai Ye ${ }^{\mathrm{a}}$, Hengbin $\mathrm{An}^{*, \mathrm{~b}, \mathrm{c}}$, Xinhai Xud,a \\ ${ }^{a}$ State Key Laboratory of High Performance Computing, National University of Defense \\ Technology, Changsha 410073, China \\ ${ }^{b}$ Laboratory of Computational Physics, Institute of Applied Physics and Computational \\ Mathematics, Beijing 100094, China \\ ${ }^{c}$ CAEP Software Center for High Performance Numerical Simulation, Beijing 100088, \\ China \\ ${ }^{d}$ Artificial Intelligence Research Center, National Innovation Institute of Defense \\ Technology, Beijing 100071, China.
}

\begin{abstract}
The radiation diffusion problem is a kind of time-dependent nonlinear equations. For solving the radiation diffusion equations, many linear systems are obtained in the nonlinear iterations at each time step. The cost of linear equations dominates the numerical simulation of radiation diffusion applications, such as inertial confinement fusion, etc. Usually, iterative methods are used to solve the linear systems in a real application. Moreover, the solution of the previous nonlinear iteration or the solution of the previous time step is typically used as the initial guess for solving the current linear equations. Because of the strong local character in ICF, with the advancing of nonlinear iteration and time step, the solution of the linear system changes dramatically in some local domain, and changes mildly or even has no change in the rest domain.

In this paper, a local character-based method is proposed to solve the linear systems of radiation diffusion problems. The proposed method consists of three steps: firstly, a local domain (algebraic domain) is constructed; secondly, the subsystem on the local domain is solved; and lastly, the whole system will be solved. Two methods are given to construct the local domain. One is based on the spatial gradient, and the other is based on the residual. Numerical tests for a two-dimensional heat conduction model problem, and two real application models, the multi-group radiation diffusion equations and the three temperature energy equations, are conducted. The test results show that the solution time for solving the linear system can be reduced dramatically by using the local
\end{abstract}

\footnotetext{
this work was funded by National Key R\&D Program of China (No. 2017YFA0603903), Science Challenge Project (No. TZ2016002) and National Natural Science Foundation of China (No. 11671051 \& No. 61872380).

${ }^{*}$ Corresponding author. Tel. +86-10-5987 2450.

Email addresses: shuaiye09@163.com (Shuai Ye), an hengbin@iapcm.ac.cn (Hengbin $\mathrm{An})$, xuxinhai@nudt.edu.cn (Xinhai Xu)
} 
character-based method.

Key words: radiation diffusion equations, linear system, local character, spatial gradient, residual

\section{Introduction}

In the simulation of astrophysics and inertial confinement fusion (ICF), it is needed to solve radiation diffusion equations. This is a strongly nonlinear system, and the discretized equations are solved by some nonlinear iterative methods, such as Newton kind of methods, Picard method, and source iteration method for multi-group radiation diffusion equations [2, 13, 17, 25]. In each nonlinear iteration, some linearized equations should be solved. In numerical simulations of ICF, usually $\mathcal{O}\left(10^{4}\right)$ time steps are needed to finish the whole simulation, and a series of linear equations $A x=b$ should be solved. The solution time for linear equations dominates the real numerical simulation cost [1, 23, 25]. It is important to design an efficient method for solving linear equations in ICF and astrophysics simulations.

Usually, the preconditioned Krylov methods [19] are used to solve the linear systems in ICF simulations. For a preconditioned Krylov method, there are four basic procedures: the construction and application of a preconditioner, the choice of an initial iterate, the computation of the next iterate and the check for stopping criterion [9, 22. Each procedure has its own effect on the iterative method. In past years, much research work has been focused on preconditioning. Among them, AMG preconditioner is the most widely used one in the simulation of radiation diffusion equations [2, 17, 23, 25].

For a series of linear systems, several techniques for choosing an efficient initial iterate have been proposed in recent years. For linear systems with the same coefficient matrix and different right-hand sides, a method for constructing an efficient initial iterate was given in [5]. Later, Chan further considered the choice of initial iterate for a series of equations with different coefficient matrices in 4. Fischer proposed another method, in which an approximate solution based upon previous solutions was used [7. In [22, a model reduction method based on proper orthogonal decomposition (POD) was used to guess a better initial iterate. The projection methods were also used in $3,6,6,10,14$. In 9,20 , a better initial guess was given by the extrapolation of previous solutions. An et al. proposed a kind of method for choosing a nonlinear initial iterate for solving two dimension three-temperature (2D 3T) heat conduction equations in 1. By solving the nonlinear system on a sub-domain where the temperature varied greatly, the method in [1] can improve the computational efficiency effectively. In [8, 12, 24, some subsystems are constructed to accelerate the convergence of nonlinear problems.

In the simulation of ICF, with time step advancing, the physical variables, such as density, pressure, temperature, etc., vary greatly in some local domain and mildly in the rest domain. This is reflected in the radiation diffusion equations, where the unknown is the temperature or radiation energy. From the 
perspective of solving linear systems from one nonlinear iteration to the next iteration, or from one time step to the next, the solution only varies greatly in some local domain while mildly in the rest. In ICF simulations, the initial iterate for solving linear equations is usually set as the previous nonlinear iterate or previous time step solution. Therefore, the solution on some local domain where the solution varies greatly has a strong influence on the convergence of an iterative method, while the solution on the rest domain has little influence on the method.

In this paper, we propose a local character-based method for solving the linear systems arising from the simulation of radiation diffusion equations. In this method, a local domain is first constructed, corresponding to a subset of the global degrees-of-freedom, on which the solution is expected to vary greatly. Then, the subsystem on the local domain is solved. At last, the whole system is solved by using the solution on the local domain. Two methods are given to construct the local domain. Specifically, the contributions of this paper are summarized as follows:

- A local character-based method for solving linear systems of radiation diffusion equations is proposed.

- Two methods are given to construct the local domain. One is based on the spatial gradient and the other is based on the residual.

- Some numerical experiments are conducted to verify the effectiveness of the proposed method. Compared with the AMG preconditioned Krylov method, the local character-based method can reduce the solution cost in most cases, and $40 \%$ cost can be saved at most.

The rest of the paper is organized as follows. The detail of the local characterbased method is presented in section 2, and also two methods for constructing the local domain are presented in this section. In section 3 , some numerical results are given and also the choice of the parameter for the local character-based method is analyzed in this section. Finally, section 4 is the summary and some remarks of the paper.

\section{The local character-based method}

In this section, the local character-based method is introduced. The basic algorithm is presented in section 2.1. Two methods for constructing the local domain are introduced in the rest. The first one, which is based on the spatial gradient, will be presented in section 2.2. The second one, which is based on the residual, will be presented in section 2.3 .

\subsection{The local character-based algorithm}

Consider a linear system resulting from implicit time propagation of discretized time dependent partial differential equations (PDEs), or the implicit 
linearization of a nonlinear system of PDEs:

$$
A x=b, \quad A \in \mathbb{R}^{N \times N}, \quad x, b \in \mathbb{R}^{N},
$$

where

$$
A=\left(\begin{array}{cccc}
a_{11} & a_{12} & \cdots & a_{1 N} \\
a_{21} & a_{22} & \cdots & a_{2 N} \\
\vdots & \vdots & \ddots & \vdots \\
a_{N 1} & a_{N 2} & \cdots & a_{N N}
\end{array}\right), \quad x=\left(\begin{array}{c}
x_{1} \\
x_{2} \\
\vdots \\
x_{N}
\end{array}\right), \quad b=\left(\begin{array}{c}
b_{1} \\
b_{2} \\
\vdots \\
b_{N}
\end{array}\right) .
$$

Eq (1) is usually obtained from some real applications, such as the application of radiation diffusion problem, etc. For solving Eq (1), an iterative method, particularly a Krylov method is often used. Because the linear system is obtained in the nonlinear iteration on some time steps, an effective initial iterate $x^{(0)}$, which is the solution of the last nonlinear iterate or the solution at last time step, is used. In the simulation process of ICF, the temperature and radiation energy only varies greatly in some local domain and varies mildly in the rest domain. Therefore, if an iterative method is used to solve Eq (1), only some components of the iterate will change greatly from the initial iterate $x^{(0)}$ to the converged solution.

For purpose of clarity, in the following we introduce some notations. Let $\Omega=\{1,2, \ldots, N\}$ be the index set of all components (degrees-of-freedom) of the linear system (1). The linear system considered in this paper is discretized from a partial differential equations, each component corresponds to one spatial point (for example, a node of the grid) in the computing domain of PDEs. Therefore, a component will also be called a point in the following discussion. $\Omega$ can also be regarded as an algebraic "domain". Assume that, from the initial iterate $x^{(0)}$ to the converged iterate, the solution varies greatly at $K$ components. For convenience, the index set of these $K$ components is denoted as $\Omega_{\text {local }}=$ $\left\{i_{1}, i_{2}, \ldots, i_{K}\right\}$. Each component in $\Omega_{\text {local }}$ is also called a bad point. $\Omega_{\text {local }}$ is called a local domain in the following. Let $\bar{\Omega}_{\text {local }}=\Omega \backslash \Omega_{\text {local }}$. Based on the partition

$$
\Omega=\Omega_{\text {local }} \cup \bar{\Omega}_{\text {local }},
$$

the linear system (1) can be partitioned as

$$
\left(\begin{array}{cc}
B & E \\
F & C
\end{array}\right)\left(\begin{array}{l}
x_{B} \\
x_{C}
\end{array}\right)=\left(\begin{array}{l}
b_{B} \\
b_{C}
\end{array}\right),
$$

where

$$
x_{B}=\left(\begin{array}{c}
x_{i_{1}} \\
x_{i_{2}} \\
\vdots \\
x_{i_{K}}
\end{array}\right), \quad b_{B}=\left(\begin{array}{c}
b_{i_{1}} \\
b_{i_{2}} \\
\vdots \\
b_{i_{K}}
\end{array}\right)
$$


and

$$
\left(\begin{array}{cc}
B & E \\
F & C
\end{array}\right)=Q^{T} A Q, \quad\left(\begin{array}{c}
x_{B} \\
x_{C}
\end{array}\right)=Q^{T} x, \quad\left(\begin{array}{c}
b_{B} \\
b_{C}
\end{array}\right)=Q^{T} b .
$$

Here $Q \in \mathbb{R}^{n \times n}$ is a permutation matrix obtained by exchanging the rows $l$ and $i_{l}(l=1,2, \ldots, K)$ of the identity matrix $I \in \mathbb{R}^{n \times n}$.

In Eq (1), $x_{B}$ is expected to change greatly from the initial iterate to the converged solution, while $x_{C}$ is expected to change mildly from the initial iterate to the converged solution. In the local character-based method, first the subsystem

$$
B x_{B}=b_{B}-E x_{C}^{(0)}
$$

will be solved, and the solution $\bar{x}_{B}$ will be obtained. Then

$$
\tilde{x}=Q\left(\begin{array}{c}
\bar{x}_{B} \\
x_{C}^{(0)}
\end{array}\right)
$$

will be used as an initial iterate to solve the whole system (1) iteratively. The specific local character-based algorithm is described by Algorithm 1 .

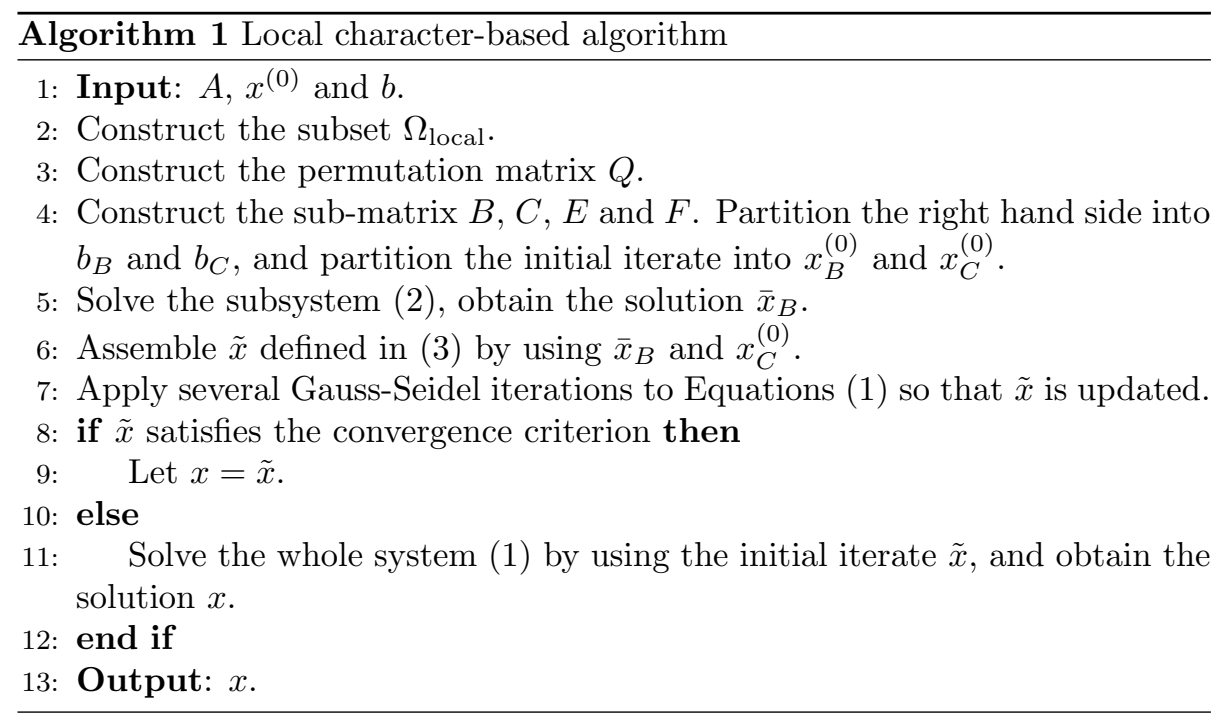

In Algorithm 1, there are two key ingredients: the first one is the construction of the subset $\Omega_{\text {local }}$ in Line 2 ; the second one is the solution of the local subsystem in Line 5 . The construction of $\Omega_{\text {local }}$ is the base of the algorithm. By $\Omega_{\text {local }}$, the whole system can be partitioned into two subsystems, and particularly the local subsystem is defined. By solving the local subsystem, a solution on $\Omega_{\text {local }}$ is obtained. After assembling the whole solution $\tilde{x}$ as the initial iterate, the whole system will be solved by an iterative method. Because the solution on $\bar{\Omega}_{\text {local }}$ is 
expected to change mildly, $x_{C}^{(0)}$ is expected to be very near to the final solution. Therefore $\tilde{x}$ will be very near to the solution. Actually, in some of our numerical cases, the residual corresponding to $\tilde{x}$ is so small that the convergence criteria is satisfied, and $\tilde{x}$ is a final solution.

The construction of the local domain, or the subset $\Omega_{\text {local }}$, plays a key role in Algorithm 1. In the following, two methods will be introduced to construct the local domain.

\subsection{Gradient-based local domain construction}

The linear system (1) is discretized from a partial differential equations (such as radiation diffusion equations). For the solution of a partial differential equations, its gradient can be defined. For example, if $T^{n}(x, y)$ is a solution of a two dimensional radiation diffusion equation at time step $n$, then the gradient of $T^{n}(x, y)$ is defined as $\nabla T^{n}(x, y)=\left(\partial T^{n} / \partial x, \partial T^{n} / \partial y\right)$. The norm of the gradient, $\left\|\nabla T^{n}(x, y)\right\|$, can be used to predict the variation of the solution from time step $n$ to time step $n+1$.

For the linear system (1), the similar norm of the gradient can be defined, which can be used to construct the local domain $\Omega_{\text {local }}$. For a point $i$, if the norm of the gradient of the initial iterate is large at this point, then it is likely that the solution may change greatly around this point, which will be considered as an element for the local domain $\Omega_{\text {local }}$. Specifically, for a given row $i$ of the matrix $A$, the non-zero entries of this row reflect the adjacency relationship of the point $i$ to other points. Therefore, the gradient $g_{i}$ at point $i$ (component $i$ ) is defined by

$$
g_{i}=\sum_{\substack{1 \leq j \leq N \\ a_{i j} \neq 0}}\left|x_{i}^{(0)}-x_{j}^{(0)}\right| .
$$

Note that this definition is not the exact gradient of a field (such as temperature, density, etc.) in two-dimensional or three-dimensional space, but just a mimicry. More precisely, this is very similar to the $l_{1}$ norm of the gradient at each point $i$. For example, if $A$ is obtained by the five-point difference scheme, then the summation in (4) includes four terms, as shown in Fig. 11 in which $p$ and $q$ are used as indices for cells. By definition in (4), the gradient of cell $(p, q)$ is

$$
\begin{aligned}
g_{p, q} & =\left|g_{p-\frac{1}{2}, q}\right|+\left|g_{p+\frac{1}{2}, q}\right|+\left|g_{p, q-\frac{1}{2}}\right|+\left|g_{p, q+\frac{1}{2}}\right| \\
& =\left|x_{p-1, q}-x_{p, q}\right|+\left|x_{p+1, q}-x_{p, q}\right|+\left|x_{p, q-1}-x_{p, q}\right|+\left|x_{p, q+1}-x_{p, q}\right| .
\end{aligned}
$$

Based on the definition of the gradient (4), the local domain can be constructed. For point $i$, if

$$
g_{i}>\alpha \max _{1 \leq j \leq N} g_{j},
$$

then $i$ will be a member of the local domain index set $\Omega_{\text {local }}$. Here $\alpha$ is a prescribed parameter. The specific algorithm is shown in Algorithm 2. 


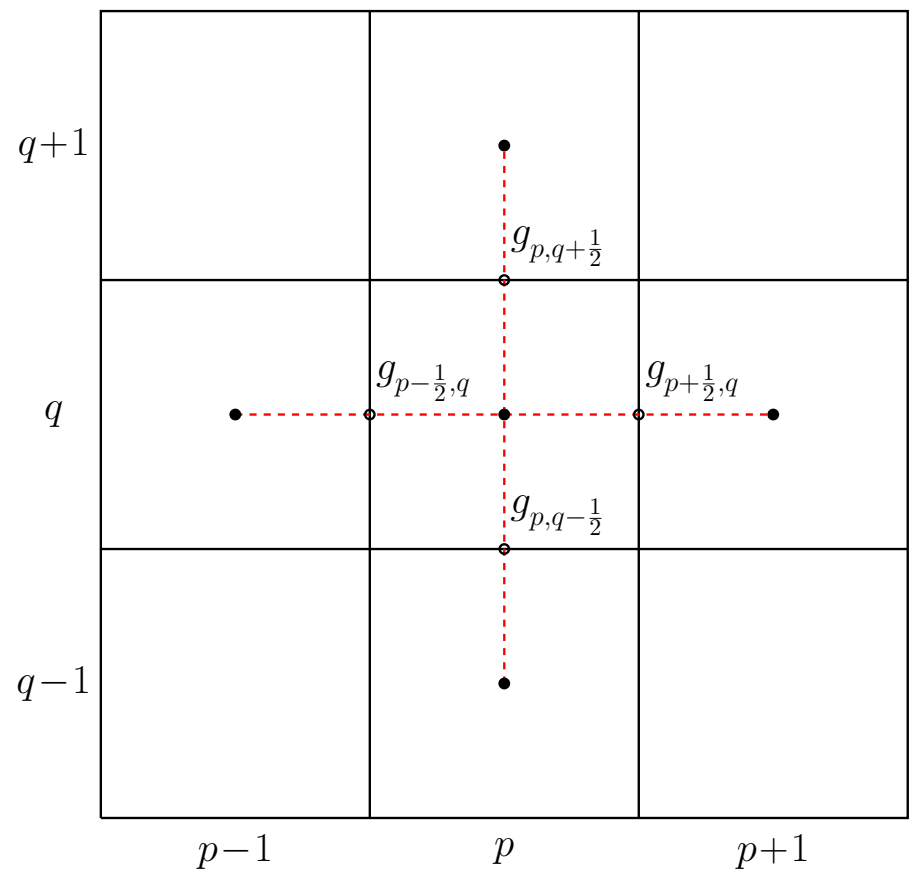

Figure 1: Gradient for five-point scheme.

\subsection{Residual-based local domain construction}

The second method for constructing the local domain is based on the residual of the linear system. The method consists of two steps. First find the components with large residual, and the bad points set, or the local domain, $\Omega_{\text {local }}$, will be constructed. Second consider the influence of the bad points on other degrees-of-freedom, and the set of bad points then extended to include other degrees-of-freedom on which the solution is also likely to change.

\subsubsection{Finding bad points}

To iteratively solve the linear system (1), a commonly used convergence criteria is given by

$$
\left\|b-A x^{(k)}\right\|_{2} \leq \epsilon\|b\|_{2},
$$

where $\epsilon$ is a prescribed tolerance and $x^{(k)}$ is an iterative solution. Since

$$
\left\|b-A x^{(k)}\right\|_{2} \leq \sqrt{N}\left\|b-A x^{(k)}\right\|_{\infty},
$$

the condition (5) will be satisfied if

$$
\left\|b-A x^{(k)}\right\|_{\infty} \leq \frac{\epsilon\|b\|_{2}}{\sqrt{N}},
$$




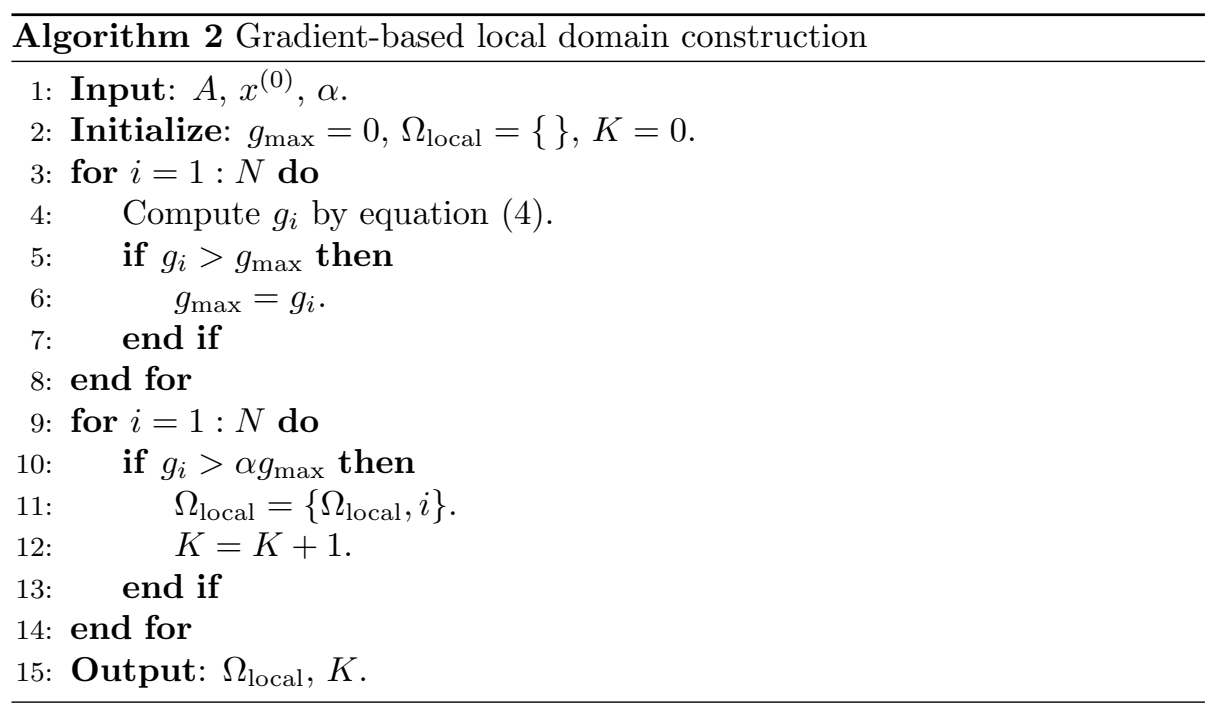

or

$$
\max _{i}\left|b_{i}-\sum_{j=1}^{N} a_{i, j} x_{j}^{(k)}\right| \leq \frac{\epsilon\|b\|_{2}}{\sqrt{N}},
$$

which is equivalent to

$$
\left|b_{i}-\sum_{j=1}^{N} a_{i, j} x_{j}^{(k)}\right| \leq \frac{\epsilon\|b\|_{2}}{\sqrt{N}}, \quad i=1,2, \ldots, N .
$$

Now, by the initial iterate $x^{(0)}$ and the convergence criteria tolerance $\epsilon$, a component $i$ is defined as a bad point if

$$
\left|b_{i}-\sum_{j=1}^{N} a_{i, j} x_{j}^{(0)}\right|>\frac{\epsilon\|b\|_{2}}{\sqrt{N}}
$$

Specifically, the initial local domain $\Omega_{\text {local }}$ is defined by

$$
\Omega_{\text {local }}=\left\{i: i=1,2, \ldots, N ;\left|b_{i}-\sum_{j=1}^{N} a_{i, j} x_{j}^{(0)}\right|>\frac{\epsilon\|b\|_{2}}{\sqrt{N}}\right\} .
$$

\subsubsection{Expanding the local domain}

When the local subsystem of the bad points is solved, the solution at the bad points is changed. This then has further influence on points adjacent to the bad points. Therefore, the set of the bad points will further be extended. That 
is, the subset of the bad points (local domain) obtained by (6) will further be expanded.

As discussed in Subsection 2.1. assume that $\bar{x}_{B}$ is the solution of the local domain subsystem, and let $\tilde{x}$ defined by (3). The $j$-th residual corresponding to $x^{(0)}$ and $\tilde{x}$ are given, respectively, as

$$
r_{j}^{(0)}=b_{j}-\sum_{i=1}^{N} a_{j, i} x_{i}^{(0)}, \quad \text { and } \quad \tilde{r}_{j}=b_{j}-\sum_{i=1}^{N} a_{j, i} \tilde{x}_{i} .
$$

By the residuals, the influence of the local domain to the point $j$ can be given.

Definition 2.1. Assume that $\Omega_{\text {local }}$ is a local domain. For one point $j \in \bar{\Omega}_{\text {local }}$, let $r_{j}^{(0)}$ be the residual at point $j$ with the initial iterate, and let $\tilde{r}_{j}$ be the residual at point $j$ after solving the local domain subsystem. If $\left|r_{j}^{(0)}\right| \leq \frac{\epsilon\|b\|_{2}}{\sqrt{N}}$ and $\left|\tilde{r}_{j}\right|>$ $\frac{\epsilon\|b\|_{2}}{\sqrt{N}}$, then the local domain $\Omega_{\text {local }}$ has influence on the point $j$; otherwise, the local domain $\Omega_{\text {local }}$ has no influence on the point $j$.

It is easy to see that

$$
\begin{aligned}
\tilde{r}_{j} & =b_{j}-\sum_{i=1}^{N} a_{j, i} \tilde{x}_{i} \\
& =b_{j}-\sum_{l=1}^{K} a_{j, i_{l}} \tilde{x}_{i_{l}}-\sum_{l=K+1}^{N} a_{j, i_{l}} \tilde{x}_{i_{l}} \\
& =b_{j}-\sum_{l=1}^{K} a_{j, i_{l}} \bar{x}_{l}-\sum_{l=K+1}^{N} a_{j, i_{l}} x_{i_{l}}^{(0)} \\
& =b_{j}-\sum_{l=1}^{K} a_{j, i_{l}}\left(\bar{x}_{l}-x_{i_{l}}^{(0)}\right)-\sum_{l=1}^{N} a_{j, i_{l}} x_{i_{l}}^{(0)} \\
& =r_{j}^{0}-\sum_{l=1}^{K} a_{j, i_{l}}\left(\bar{x}_{l}-x_{i_{l}}^{(0)}\right) .
\end{aligned}
$$

Assume that

$$
\left|\bar{x}_{l}-x_{i_{l}}^{(0)}\right| \leq\left|x_{i_{l}}^{(0)}\right|, \quad l=1,2, \ldots, K,
$$

which means the relative variation of the solution at all the bad points is less than one (This is satisfied in our numerical tests). Then it is easy to see that

$$
\left|\tilde{r}_{j}\right| \leq\left|r_{j}^{(0)}\right|+\sum_{l=1}^{K}\left|a_{j, i_{l}} x_{i_{l}}^{(0)}\right|, \quad j=1,2, \ldots, N .
$$

This inequality shows that if

$$
\left|r_{j}^{(0)}\right|+\sum_{l=1}^{K}\left|a_{j, i_{l}} x_{i_{l}}^{(0)}\right| \leq \frac{\epsilon\|b\|_{2}}{\sqrt{N}},
$$


then the local domain will have not influence on point $j$ (by Definition 2.1). Otherwise, the local domain will be expected to have influence on point $j$. In this case, the local domain will be extended. Specifically, assume that $j \in \bar{\Omega}_{\text {local }}$ is a neighbor of $\Omega_{\text {local }}$. If

$$
\left|r_{j}^{(0)}\right|+\sum_{l=1}^{K}\left|a_{j, i_{l}} x_{i_{l}}^{(0)}\right|>\frac{\epsilon\|b\|_{2}}{\sqrt{N}},
$$

then $j$ will be incorporated into the local domain subset $\Omega_{\text {local }}$.

Note that the extension process can be implemented several times until the size of the subset $\Omega_{\text {local }}$ no longer increases. In practice, a few times of expanding is enough. The specific process is given in Algorithm 3 .

In Algorithm 3, two steps are used to construct the local domain $\Omega_{\text {local }}$. The first step is to construct $\Omega_{\text {local }}$ by the component residuals, and those components with large residuals are incorporated into $\Omega_{\text {local }}$. The second step is to extend $\Omega_{\text {local }}$ by at most $E_{\text {max }}$ iterations. In each expanding iteration, some neighbors of $\Omega_{\text {local }}$ are further incorporated into $\Omega_{\text {local }}$.

Example 1. The following example shows the specific implementation of Algorithm 3. Consider a $9 \times 9$ linear system with the coefficient matrix given by

$$
A=\left(\begin{array}{ccccccccccc}
1 & -\frac{1}{2} & & & & & & & \\
-\frac{1}{2} & 1 & -\frac{1}{3} & & & & & & \\
& -\frac{1}{3} & 1 & -\frac{1}{4} & & & & & \\
& & -\frac{1}{4} & 1 & -\frac{1}{5} & & & & \\
& & & -\frac{1}{5} & 1 & -\frac{1}{6} & & & \\
& & & & -\frac{1}{6} & 1 & -\frac{1}{7} & & \\
& & & & & -\frac{1}{7} & 1 & -\frac{1}{8} & \\
& & & & & & -\frac{1}{8} & 1 & -\frac{1}{9} \\
& & & & & & & -\frac{1}{9} & 1
\end{array}\right)
$$

The solution and the initial iterate are set, respectively, as

$$
x=\left(\begin{array}{c}
1 e-1 \\
1 e-2 \\
1 e-3 \\
1 e-4 \\
1 e-5 \\
1 e-6 \\
1 e-7 \\
1 e-8 \\
1 e-9
\end{array}\right), \quad x^{(0)}=\left(\begin{array}{c}
1 \\
1 e-1 \\
1.001 e-3 \\
1.001 e-4 \\
1.001 e-5 \\
1.001 e-6 \\
1.001 e-7 \\
1.001 e-8 \\
1.001 e-9
\end{array}\right) .
$$

The right hand side is set to $b=A x$.

Consider the application of Algorithm 3 to this linear system. The convergence criterion is set to $\epsilon=1 e-5$, and the parameter for maximal number of 


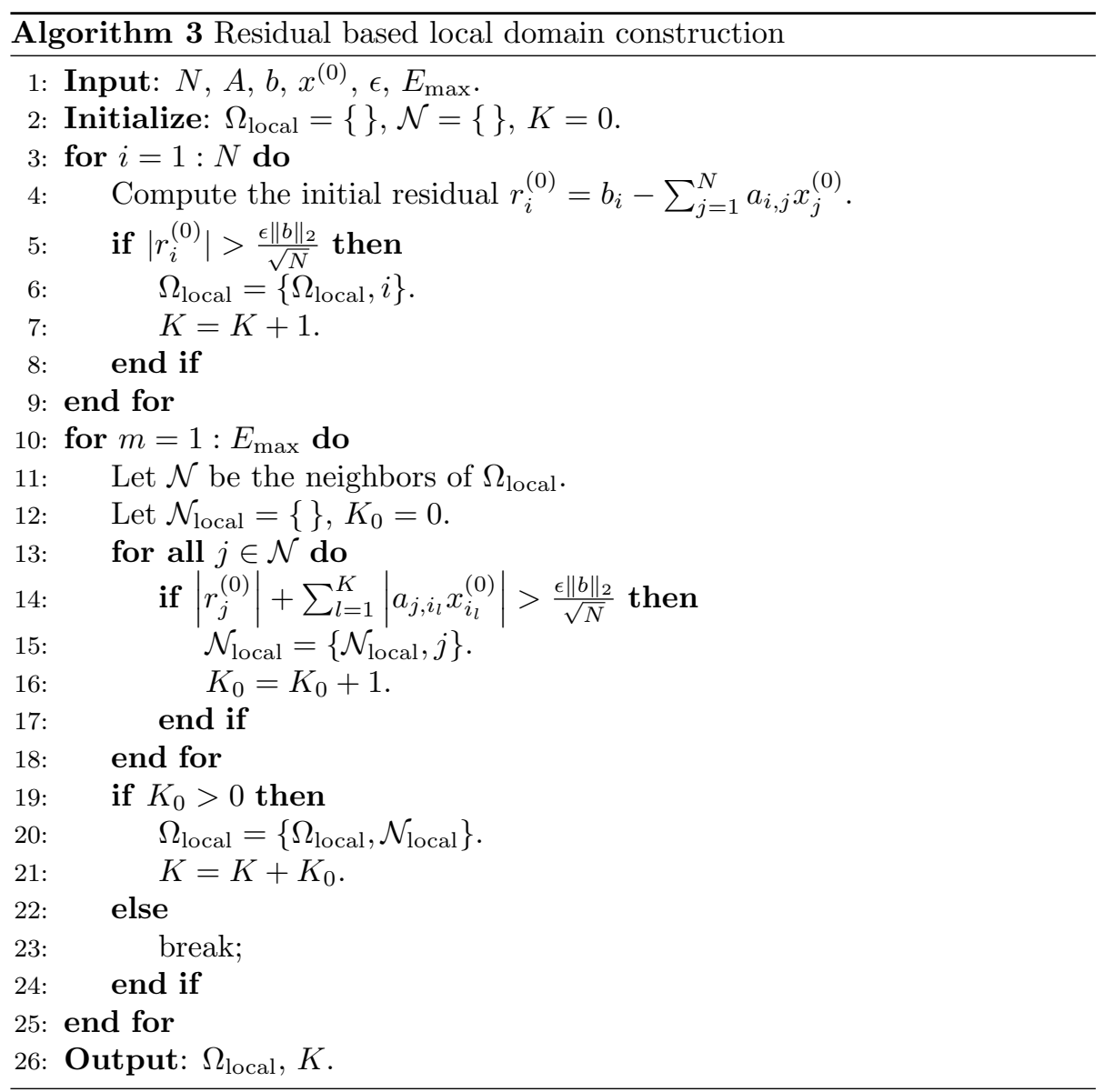


extension is set to $E_{\max }=6$. It is easy to check that $\epsilon\|b\|_{2} / \sqrt{N}=3.44 e-7$. The initial residual is

$$
r^{(0)}=b-A x^{(0)}=\left(\begin{array}{c}
-8.55 e-1 \\
3.66 e-1 \\
3.00 e-2 \\
1.52 e-7 \\
1.02 e-8 \\
6.81 e-10 \\
4.41 e-11 \\
2.61 e-12 \\
1.11 e-13
\end{array}\right)
$$

In the first step of Algorithm 3, points 1, 2 and 3 are marked as the bad points, i.e., $\Omega_{\text {local }}=\{1,2,3\}$. In the second step of Algorithm 3, $\Omega_{\text {local }}$ is further extended. The loop of extension process is implemented 4 times. After each loop, the main results are listed in the following table. In the fourth iteration, $\mathcal{N}=\{7\}$ and $\left|r_{7}^{(0)}\right|+\left|a_{76} \cdot x_{6}^{(0)}\right|=1.430 e-7<\epsilon\|b\|_{2} / \sqrt{N}=3.44 e-7$, therefore, $K_{0}=0$ and no point will be incorporated into $\Omega_{\text {local }}$. The loop will break.

Table 1: The extension process of $\Omega_{\text {local }}$.

\begin{tabular}{cccccc}
\hline$m$ & $\mathcal{N}$ & $\mathcal{N}_{\text {local }}$ & $\Omega_{\text {local }}$ & $K$ & $\left|r_{j}^{(0)}\right|+\sum_{l=1}^{K}\left|a_{j, i_{l}} x_{i_{l}}^{(0)}\right|$ \\
\hline 1 & $\{4\}$ & $\{4\}$ & $\{1,2,3,4\}$ & 4 & $\left|r_{4}^{(0)}\right|+\left|a_{43} \cdot x_{3}^{(0)}\right|=2.504 e-4$ \\
\hline 2 & $\{5\}$ & $\{5\}$ & $\{1,2,3,4,5\}$ & 5 & $\left|r_{5}^{(0)}\right|+\left|a_{54} \cdot x_{4}^{(0)}\right|=2.003 e-5$ \\
\hline 3 & $\{6\}$ & $\{6\}$ & $\{1,2,3,4,5,6\}$ & 6 & $\left|r_{6}^{(0)}\right|+\left|a_{65} \cdot x_{5}^{(0)}\right|=1.669 e-6$ \\
\hline 4 & $\{7\}$ & $\emptyset$ & $\{1,2,3,4,5,6\}$ & 6 & $\left|r_{7}^{(0)}\right|+\left|a_{76} \cdot x_{6}^{(0)}\right|=1.430 e-7$ \\
\hline
\end{tabular}

\subsection{Parallelization of the local character-based methods}

For parallel computing, assume that $p$ processors are used. For simplicity, assume that $N=\bar{N} \times p$, where $\bar{N}$ is an integer. The matrix $A$ is partitioned by rows, that is

$$
A=\left[\begin{array}{llll}
A_{\underline{1}}^{T} & A_{\underline{2}}^{T} & \cdots & A_{\underline{p}}^{T}
\end{array}\right]^{T},
$$

where $A_{\underline{l}}$ is a $\bar{N} \times N$ matrix, $l=1,2, \ldots, p$. The solution and right-hand side are partitioned correspondingly

$$
x=\left[\begin{array}{llll}
x_{\underline{1}}^{T} & x_{\underline{2}}^{T} & \cdots & x_{\underline{p}}^{T}
\end{array}\right]^{T}, \quad b=\left[\begin{array}{llll}
b_{1}^{T} & b_{\underline{2}}^{T} & \cdots & b_{\underline{p}}^{T}
\end{array}\right]^{T},
$$

where $x_{\underline{l}}$ and $b_{\underline{l}}$ are $\bar{N}$ vectors, $l=1,2, \ldots, p$. For convenience, let

$$
\mathcal{I}_{\underline{l}}=\{(l-1) \bar{N}, \ldots, l \bar{N}\}
$$


be the component index set of the $l$-th processor, $l=1,2, \ldots, p$.

The key work in the parallel version of the algorithm is to construct the local domain $\Omega_{\text {local }}$. When the local domain is constructed, the local domain subsystem can be obtained easily. For solving the local domain subsystem, any parallel solution method can be used. In the following, Algorithm 4 and Algorithm 5 are given for the gradient based and residual based local domain construction in parallel computing case.

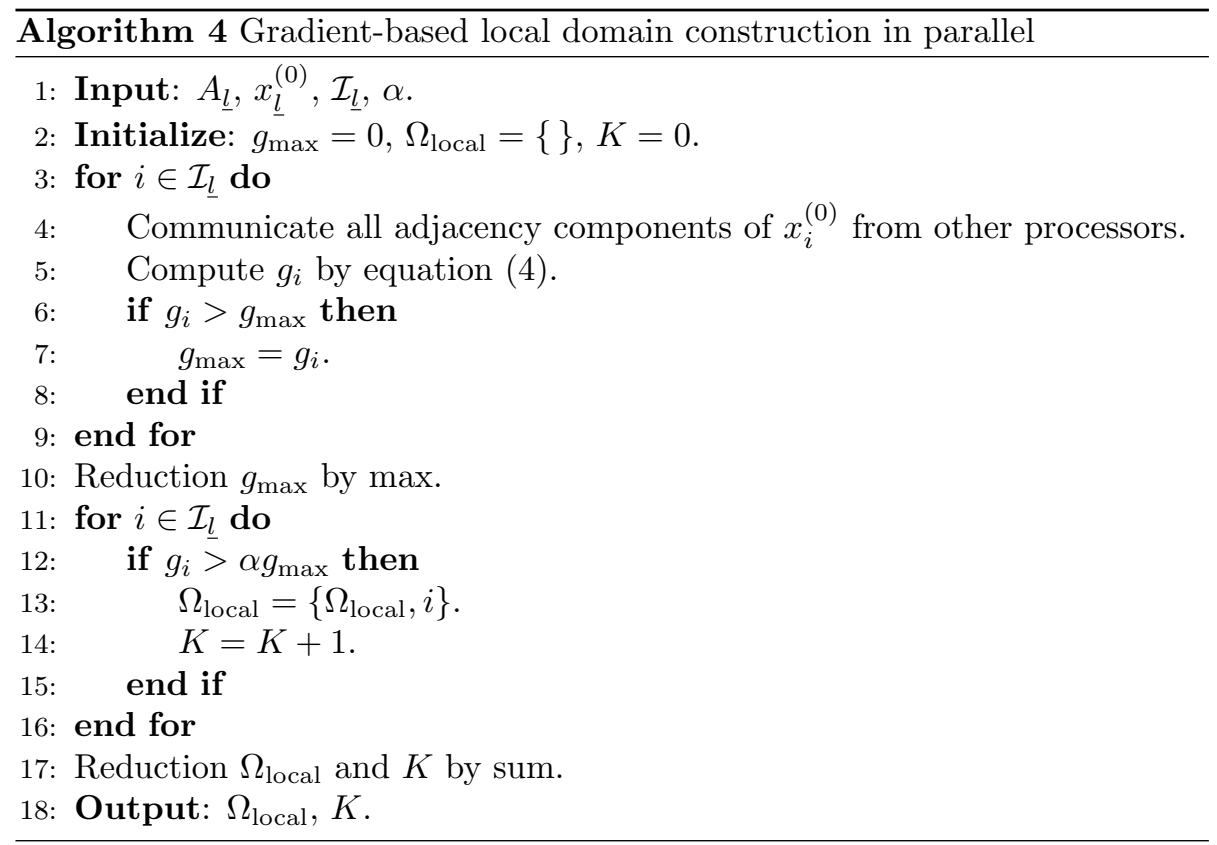

Compared to Algorithm 2, some communications should be implemented for parallel case, which is shown in Line 4, 10, and 17 in Algorithm 4

Compared to Algorithm 3 , it is needed to have some communication in Line 4, 11, 16, and 25 in Algorithm 5.

It should be noted that for both Algorithm 4 and Algorithm 5, some global reductions are needed to be implemented. For example, the computation of the right hand side norm, etc.

\section{Numerical Results}

In this section, one test model and two suites of real application linear systems are used to test the effectiveness of the local character-based method. The test model is a two-dimensional nonlinear heat conduction equation. The two suites of real application linear systems are respectively the multi-group radiation diffusion equations and the three temperature energy equations.

All experiments are carried out on an E5-2620 CPU, which is clocked at 2.10 GHz and has a 16 GB memory. E5-2620 has a total of 12 cores on two sockets. 


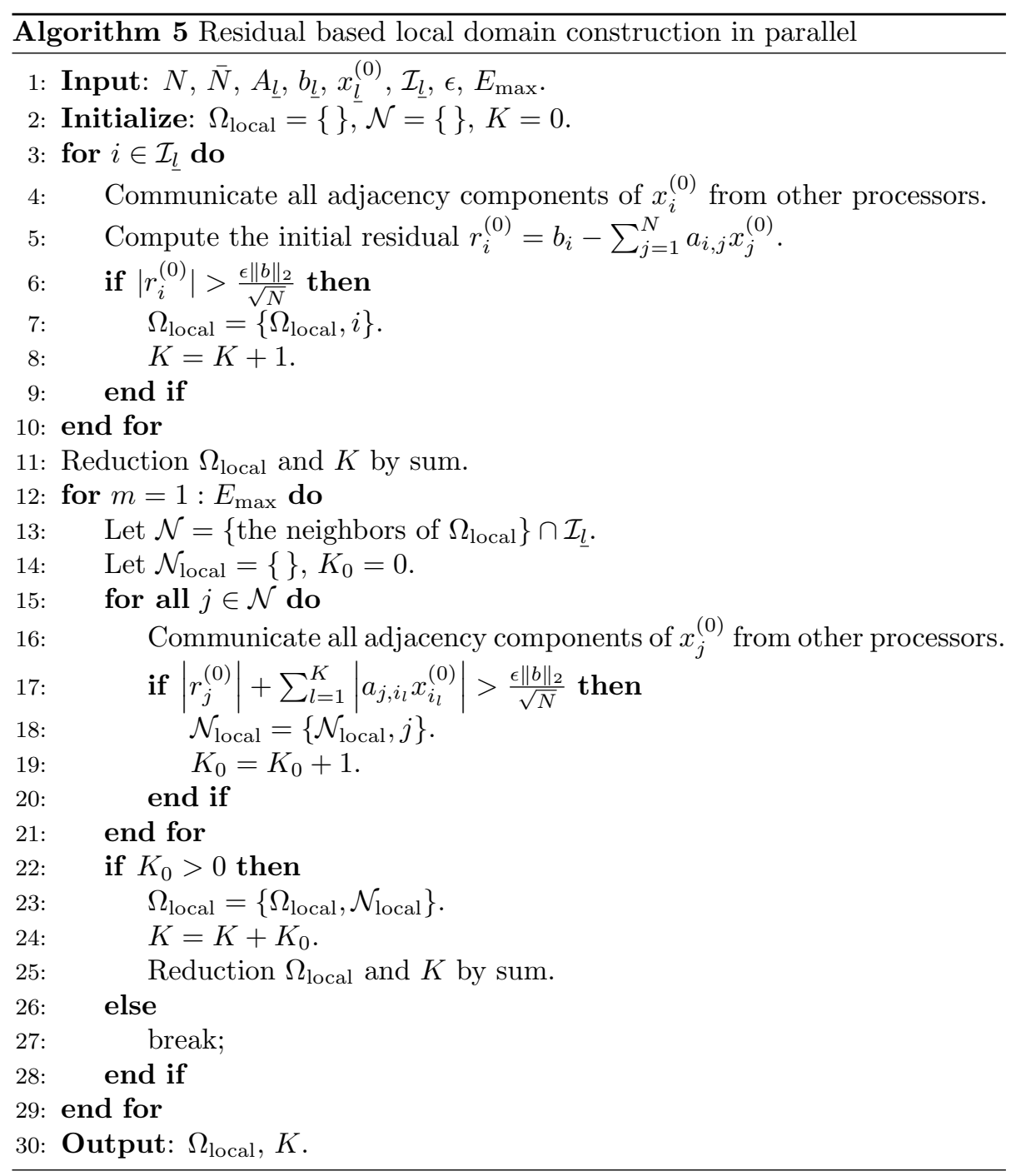


The local character-based method is compared with the typical solution method. In the test, the compared method is a BoomerAMG preconditioned GMRES method. For BoomerAMG, Falgout coarsening (a combination of CLJP and the classical Ruge-Stuben coarsening) and "classical" interpolation [18 are used, the maximal number of levels is set to 8, and one symmetric Gauss-Seidel iteration is used for both pre-relaxation and post-relaxation. The maximal GMRES iteration number is set to 80, and the Krylov dimension is 40 .

In the local character-based method, the local domain subsystem is solved by the BoomerAMG preconditioned GMRES method with completely the same parameters for solving the global system. One Gauss-Seidel iteration is applied to smooth $\tilde{x}$. In the local character-based methods, the parameters $\alpha$ in Algorithm 2 and $E_{\max }$ in Algorithm 3 are predefined by users, and some numerical analysis is given for different $\alpha$ and $E_{\max }$.

For the local character-based method, after solving the sub-domain system, the assembled solution $\tilde{x}$ in (3) satisfies the convergence criteria in most of the tests. Therefore, no further iteration is implemented for the global system in most cases.

For the purpose of presenting the results, the following notations are used to represent the specific methods:

- METHOD0: BoomerAMG preconditioned GMRES method.

- METHOD1: Local character-based method in which the local domain is constructed by Algorithm 2

- METHOD2: Local character-based method in which the local domain is constructed by Algorithm 3

Furthermore, the following notations will be used to report the results.

- $N$ : the scale of the global linear system.

- $K$ : the scale of the sub-domain linear system in the local character-based method.

- $\eta=\frac{K}{N} \times 100 \%$ : percentage of the scale of the sub-domain linear system to the scale of the global system.

- $\mathrm{CPU}_{\text {total }}^{\text {glb-meth }}$ : CPU time for solving the linear system by BoomerAMG preconditioned GMRES method.

- $\mathrm{CPU}_{\text {total }}^{\text {loc-meth: }}$ CPU time for solving the linear system by the local characterbased method.

- $\mathrm{CPU}_{\text {loc-constr: }} \mathrm{CPU}$ time for constructing the local domain in the local character-based method.

- $\mathrm{CPU}_{\text {loc-solv }}$ : $\mathrm{CPU}$ time for solving the sub-domain system in the local character-based method.

- $S_{\mathrm{CPU}}$ : the speedup of CPU time for solving the linear system by the local character-based method to BoomerAMG preconditioned GMRES method. 


\subsection{Test of two-dimensional heat conduction equation}

Consider the following two-dimensional (2-D) heat conduction equations:

$$
\begin{cases}\frac{\partial T}{\partial t}=\nabla \cdot(\kappa(T) \nabla T), & 0<x<1,0<y<1,0<t<\infty \\ T(0, x, y)=T_{0}(x, y), & 0<x<1,0<y<1, t=0 \\ T(t, 0, y)=T_{l}, & x=0,0<t<\infty \\ T(t, 1, y)=T_{r}, & x=1,0<t<\infty \\ \frac{\partial T}{\partial y}=0, & y=0, y=1,0<t<\infty\end{cases}
$$

In this test, $T_{l}, T_{r}, \kappa(T)$ and $T_{0}(x, y)$ are set respectively as

$$
T_{l}=1, \quad T_{r}=1 \times 10^{-4}, \quad \kappa(T)=T^{3.5}, \quad T_{0}(x, y)=e^{-100 x} \times T_{l}+T_{r} .
$$

By using the backward Euler method to discretize in time, one obtains

$$
\frac{T^{n+1}-T^{n}}{\Delta t}=(\nabla \cdot(\kappa(T) \nabla T))^{n+1}
$$

The mesh is $99 \times 99$, and a five-point finite difference method is used to discretize in space, and a nonlinear system is obtained.

In this test, the time step size is $\Delta t=10^{-2}$. The simulation is implemented from physical time 0 to 1 , and the total number of time steps is 100 . The Picard method is used to solve the system, and the linear system in Picard iteration will be solved by METHOD0, METHOD1, or METHOD2. The parameter $\alpha$ in METHOD1 is set to $10^{-4}$, and the $E_{\max }$ in METHOD2 is set to 1 . For clarity, the time advancing and nonlinear iteration process is given in Algorithm 6

The $n$ loop in Algorithm 6 is for time advancing, and the $s$ loop is for nonlinear iteration. Eq. (9) is the Picard linearized system. The convergence tolerance for the linear system is $\epsilon=10^{-10}$, and the stopping criterion for the nonlinear Picard iteration is $\left\|T^{n+1, s+1}-T^{n+1, s}\right\|_{2}<10^{-8}$. In the computation, the previous nonlinear iterate is used as the initial guess for solving the current linear system.

First a comparison between the solutions obtained by the three different methods is conducted. Fig. 2 shows the difference in solution obtained by different methods as a function of time, given by

$e_{1}^{0}=\left\|T_{\text {METHOD } 1}-T_{\text {METHodo }}\right\|_{2} /\left\|T_{\text {METHODo }}\right\|_{2}, \quad e_{2}^{0}=\left\|T_{\text {METHOD } 2}-T_{\text {METHODo }}\right\|_{2} /\left\|T_{\text {METHODo }}\right\|_{2}$.

Here, $T_{\text {METHOD } 0}, T_{\text {METHOD } 1}$, and $T_{\text {METHOD2 }}$ are the solutions obtained by METHOD0, METHOD1, and METHOD2, respectively, at each time step. From this figure, one can see that for all 100 time steps, the maximal value of $e_{1}^{0}$ and $e_{2}^{0}$ are, respectively, $7.6 \times 10^{-9}$ and $7.0 \times 10^{-9}$. This shows that the approximation solution obtained by different methods are very close and they can be considered almost the same.

In the test, if METHOD0, METHOD1, or METHOD2 is used to solve all the linear systems for all 100 time steps, the total CPU time for solving the linear systems 

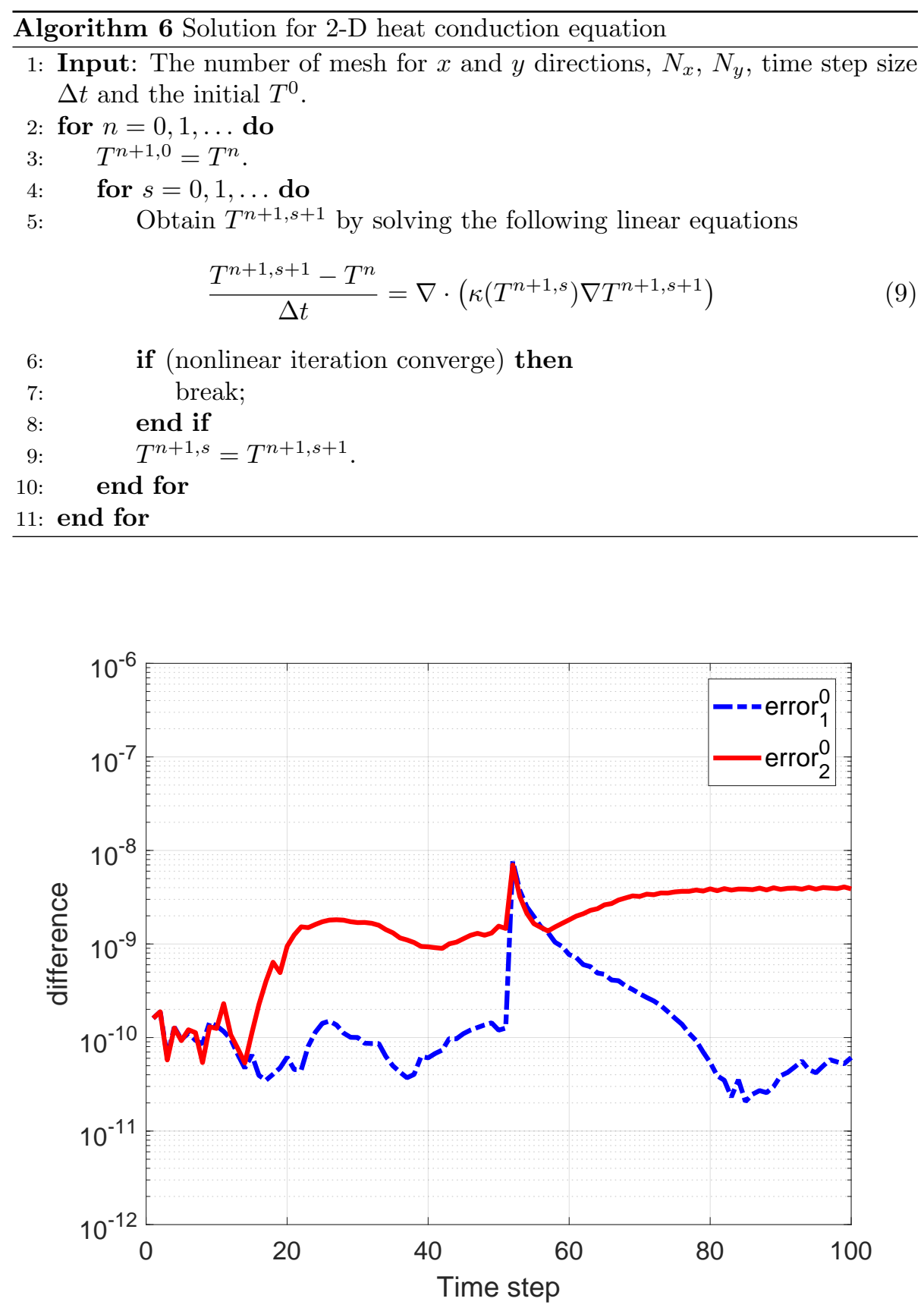

Figure 2: The differences of the solution obtained by METHOD0, METHOD1, and METHOD2 for two dimensional heat conduction problem. 
is respectively $39.19 \mathrm{~s}, 28.09 \mathrm{~s}$, and $29.76 \mathrm{~s}$. Therefore, the speedups of METHOD1 and METHOD2 to METHOD0 are about 1.40 and 1.32, respectively.

Fig. 3 shows the average speedups of the two local character-based methods at each time step. At same time, the average percentage of the scale of the sub-domain linear system to the scale of the global system, $\eta$, for two local character-based methods is shown in the figure. From this figure, one can see that both methods have the similar performance in the simulation. With time advancing, the speedup deceases, and $\eta$ increases. At later simulation time, METHOD2 performs a little better than METHOD1.

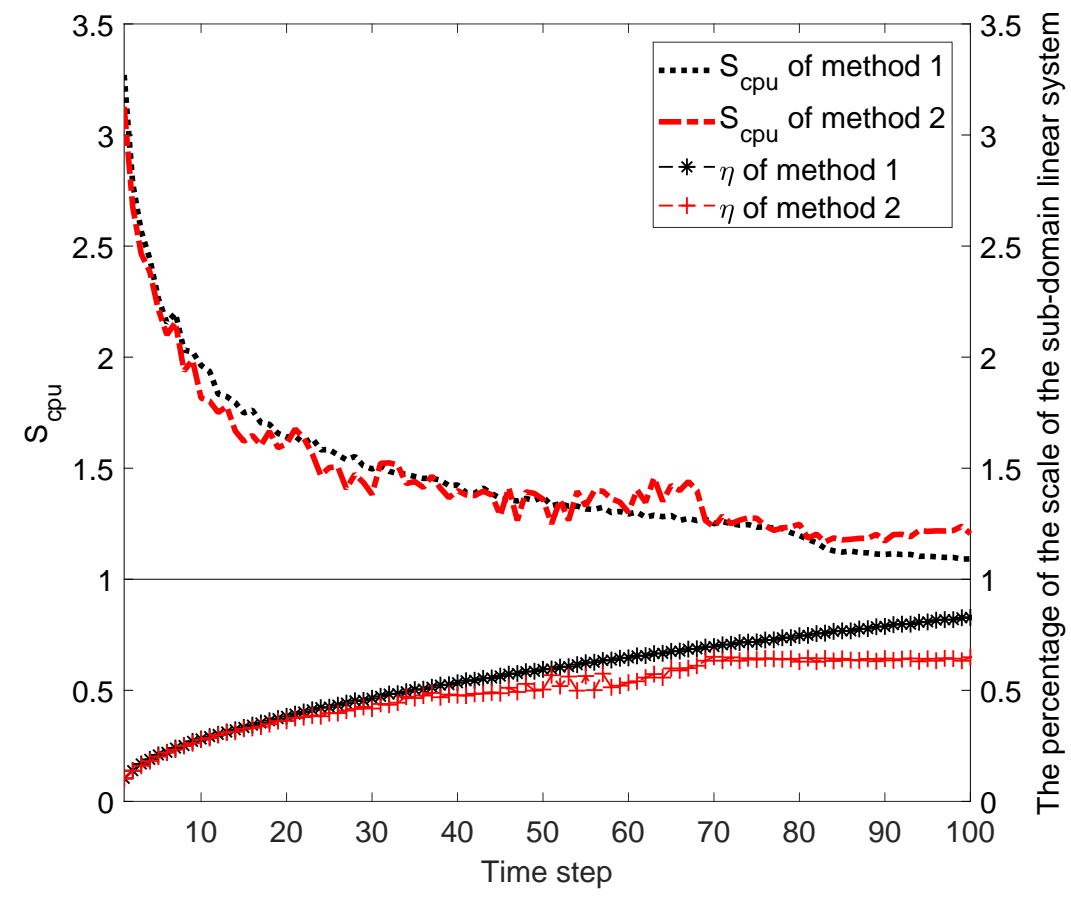

Figure 3: The average speedup and average percentage of the scale of the sub-domain linear system to the scale of the global system for two local character-based methods.

Fig. 4 shows the specific speedup and percentage of the scale of the subdomain linear system to the scale of the global system at each nonlinear iteration for the two local character-based methods. In the figure, the results at four time steps are given. From this figure, one can see that METHOD1 have a relatively stable performance at each time step. At the same time, the percentage of the sub-domain linear system is stable for all nonlinear iterations at each time. With time advancing, the performance of METHOD1 decreases. For METHOD2, one can see that it has similar performance for the early 6 nonlinear iterations at all time steps. For the later nonlinear iterations, METHOD2 performs unstable. At about three nonlinear iterations, its performance deteriorates. 

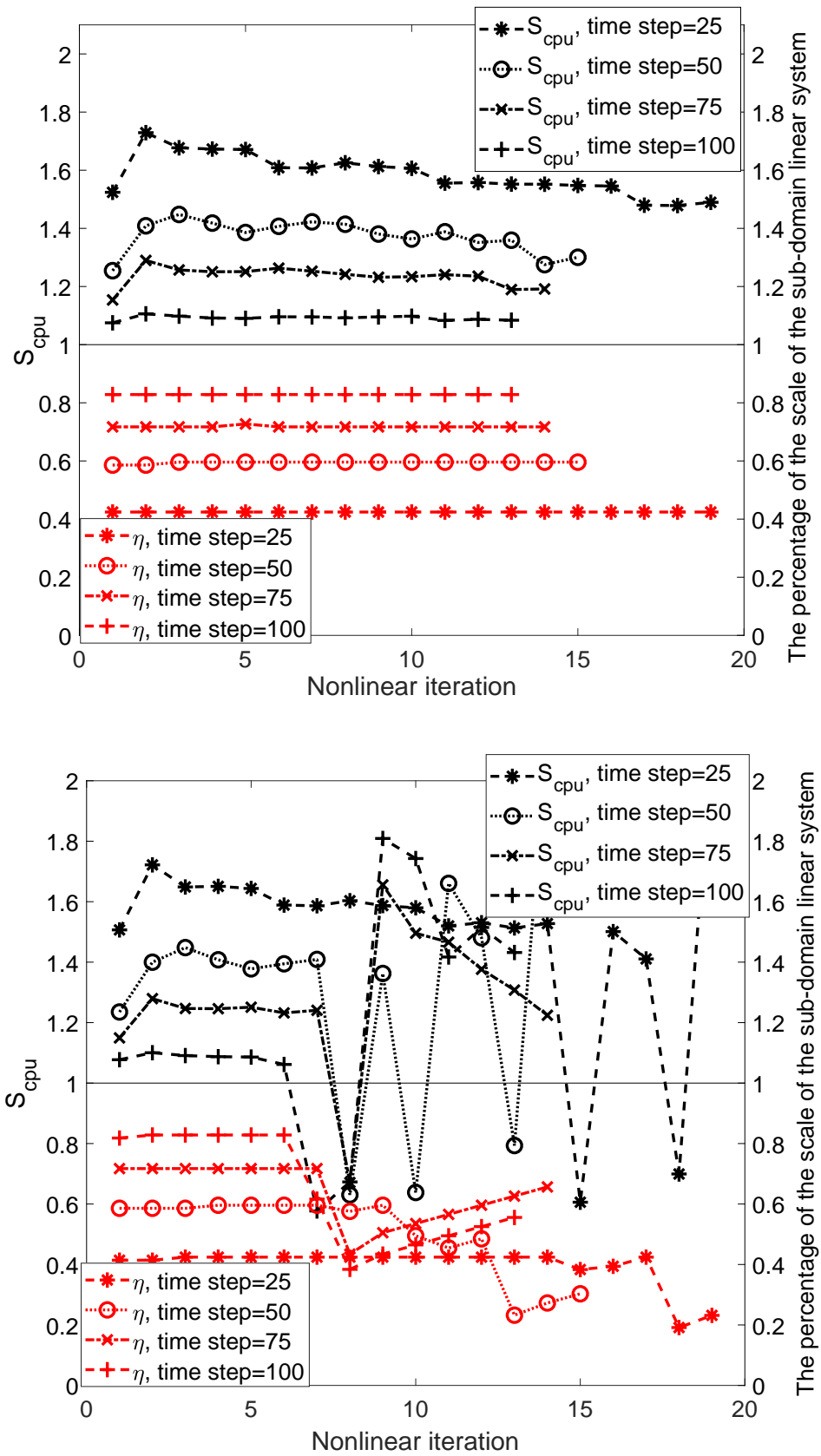

Figure 4: The percentage of the scale of the subsystem and the speedup of the local characterbased methods for solving linear systems of the 2-D heat conduction equations at time steps $25,50,75$, and 100. The upper is the result of METHOD1, the lower is the result of METHOD2. 
For 2-D heat conduction equation, the local domains determined by METHOD1 and METHOD2 are almost the same. As examples, Fig. 5 shows the specific local domain in the first nonlinear iteration at time steps $25,50,75$, and 100. At the same time, the solutions at these two time steps are shown in the figure. One see can that the local domain $\Omega_{\text {local }}^{1}$ and $\Omega_{\text {local }}^{2}$ are almost the same, and they just contain the region where the solution varies greatly. Here $\Omega_{\text {local }}^{1}$ represents the local domain obtained by Algorithm 2 and $\Omega_{\text {local }}^{2}$ represents the local domain obtained by Algorithm 3
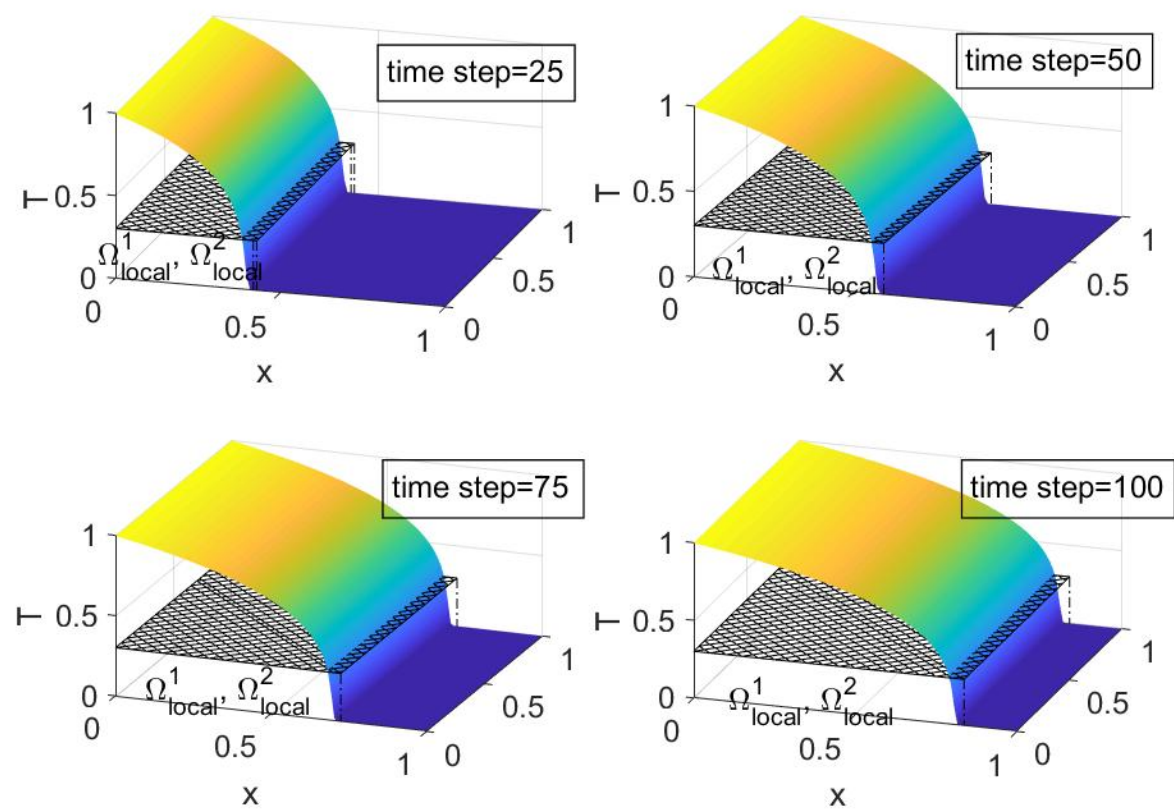

Figure 5: The local domain for two dimensional heat conduction problem at time steps 25, 50,75 , and 100 (the first nonlinear iteration).

\subsection{Scalability of the local character-based methods for the 2-D heat conduction equation}

In this section, the scalability test of the local character-based methods are done for the 2-D heat conduction equation. In the test, the scale on each processor is fixed as $512 \times 512$, and the number of processors varies from 1 to 256. For all tests, the time step size is fixed as $\Delta t=10^{-2}$, and the number of time steps is 100. The test results are listed in Table 2. In the table, the following notations are used:

- $\mathrm{CPU}_{\text {total }}$ : CPU time for solving all the linear systems in the simulation.

- $N_{\text {eq }}$ : Total number of linear equations in the simulation. 
Table 2: Scalability test of METHOD0, METHOD1, and METHOD2 for 2-D heat conduction equation

\begin{tabular}{|c|c|c|c|c|c|c|}
\hline \#cores & Method & $N$ & $\mathrm{CPU}_{\text {total }}$ & $N_{\text {eq }}$ & $\overline{\mathrm{CPU}_{\text {total }}}$ & $S_{\mathrm{CPU}}$ \\
\hline \multirow{3}{*}{1} & METHOD0 & \multirow{3}{*}{$512^{2}$} & 1087.45 & 2757 & 0.39 & - \\
\hline & METHOD1 & & 693.38 & 2757 & 0.25 & 1.56 \\
\hline & METHOD2 & & 760.71 & 2758 & 0.28 & 1.42 \\
\hline \multirow{3}{*}{4} & METHOD0 & \multirow{3}{*}{$1024^{2}$} & 2364.74 & 3281 & 0.72 & - \\
\hline & METHOD1 & & 927.42 & 3288 & 0.28 & 2.55 \\
\hline & METHOD2 & & 1148.46 & 3319 & 0.35 & 2.06 \\
\hline \multirow{3}{*}{16} & METHOD0 & \multirow{3}{*}{$2048^{2}$} & 3783.12 & 3655 & 1.04 & - \\
\hline & METHOD1 & & 1105.13 & 3770 & 0.29 & 3.42 \\
\hline & METHOD2 & & 1608.81 & 3844 & 0.42 & 2.35 \\
\hline \multirow{3}{*}{64} & METHOD0 & \multirow{3}{*}{$4096^{2}$} & 4172.75 & 3955 & 1.06 & - \\
\hline & METHOD1 & & 1100.34 & 4253 & 0.26 & 3.79 \\
\hline & METHOD2 & & 1789.89 & 4293 & 0.42 & 2.33 \\
\hline \multirow{3}{*}{256} & METHOD0 & \multirow{3}{*}{$8192^{2}$} & 4621.16 & 4052 & 1.14 & - \\
\hline & METHOD1 & & 1283.76 & 4741 & 0.27 & 3.60 \\
\hline & METHOD2 & & 2173.07 & 4676 & 0.46 & 2.13 \\
\hline
\end{tabular}

- $\overline{\mathrm{CPU}_{\text {total }}}$ : The average CPU time for solving one linear system.

- $S_{\text {CPU }}$ : The speedup of METHOD1 and METHOD2 to METHOD0 (based on the average CPU time for solving one linear system).

From Table 2, one can see that METHOD1 and METHOD2 scale better than METHOD0 by comparing the the total CPU time for solving all the linear equations, $\mathrm{CPU}_{\text {total }}$. By comparing the numbers of linear equations for different methods, we find that more linear equations are solved for METHOD1 and METHOD2 than METHOD0. By comparing the average CPU time for solving one linear equation, one can see that METHOD1 and METHOD2 perform much better than METHODO. Correspondingly, the speedups of METHOD1 and METHOD2 (for solving one linear equation) are from 1.42 to 3.79 . With the increase of the scale, the speedup increases for both METHOD1 and METHOD2.

\subsection{Test of multi-group radiation diffusion equations}

In this subsection, the local character-based method will be used to solve the multi-group radiation diffusion equations in real applications. The linear equations for the test are extracted from the radiation hydrodynamics codeLared-integration, which is used for inertial confinement fusion (ICF) study [21]. 


\subsubsection{Multi-group radiation diffusion equations}

The multi-group radiation diffusion equations are a set of $G$ coupled partial differential equations, which are given as follows:

$$
\frac{\partial E_{g}}{\partial t}=\nabla \cdot \kappa_{g} \nabla E_{g}+4 \pi \sigma_{g} B_{g}-c \sigma_{g} E_{g}, \quad g=1, \cdots, G
$$

In the equations,

- $G$ is the number of energy groups.

- $E_{g}$ is the radiation energy density of the $g$-th group.

- $\kappa_{g}$ is the radiation diffusion coefficient of the $g$-th group.

- $B_{g}$ is the integration of the Planck function of the $g$-th group.

- $\sigma_{g}$ is the cross section of the $g$-th group.

In real applications, the multi-group radiation diffusion equations are coupled to electron-ion equations, and they form a strong nonlinear system. For more details about the radiation diffusion model, see [11, 25]. We will focus on the solution for the multi-group radiation diffusion equations in this paper. Equations $(10)$ are a time-dependent nonlinear system. The backward Euler method is used to discretize in time. For solving the nonlinear system after temporal discretization, a nonlinear iteration (based on Picard-Newton linearization) is used, and in each nonlinear iteration, the source iteration method is used to solve the multi-group radiation diffusion equations [11, 16. In each source iteration, the $G$ radiation diffusion equations are decoupled, and each group radiation diffusion equation can be solved independently. By considering the time advancing, Algorithm 7 can be used to describe the main solution procedure for multi-group radiation diffusion equations.

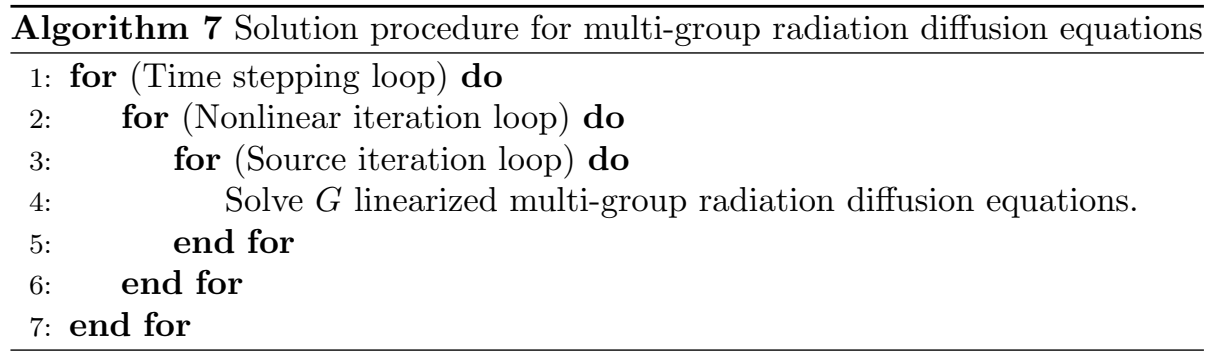

A Lagrangian method is used in the Lared-integration code and the mesh moves with the motion of the fluid. Consequently, the multi-group radiation diffusion equations are discretized on two-dimensional deforming meshes. A nine-point scheme is used to discretize the diffusion equations. 


\subsubsection{Linear systems for test}

In the test, the number of cells is 7040 . The number of groups is $G=64$, and therefore, 64 equations must be solved in each source iteration.

Linear systems are tested for all groups, in a given source iteration of a given nonlinear iteration, at time steps 1,000 and 5,000. For notation, each linear system is denoted as $\mathrm{T} n_{1} \mathrm{~N} n_{2} \mathrm{~S} n_{3} \mathrm{G} n_{4}$, which means the linear system of group $n_{4}$ in the $n_{3}$ th source iteration of $n_{2}$ th nonlinear iteration at $n_{1}$ th time step. For example, T1000N0S0G0 represents the linear system of 0-th group in 0 -th source iteration of 0 -th nonlinear iteration at time step 1,000. All the coefficient matrices of the linear system have the same sparsity pattern, shown in Fig. 6.

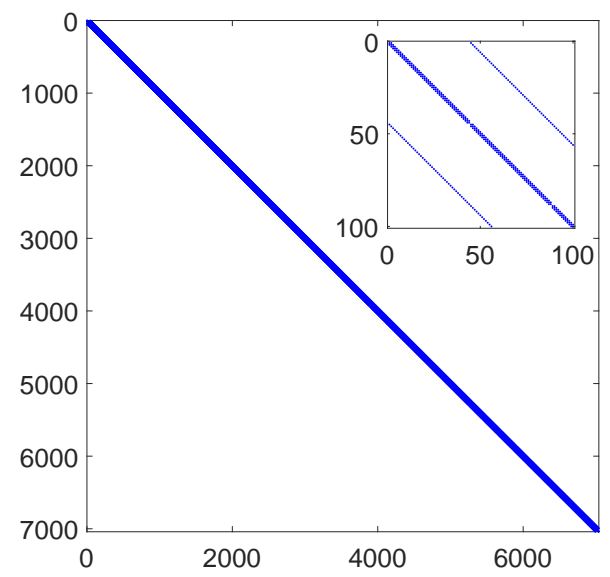

Figure 6: The sparsity pattern of the matrix of the multi-group radiation diffusion linear system.

\subsubsection{Test results}

We test a total of 128 linear systems on two time steps, i.e., T1000N0S0G0G63 and T5000N0S0G0-G63. In this test, the parameter $\alpha$ in METHOD1 is set to $10^{-10}$, and $E_{\max }$ in METHOD2 is set to 1 . The convergence tolerance $\epsilon=10^{-8}$. The number of the Gauss-Seidel iteration is one in Algorithm 1. The speedups and the percentages of the scale of the local domain are shown in Fig. 7

In Fig. 7. at 1,000th time step, the maximal speedup of METHOD1 and METHOD2 for all the 64 equations are 1.61 and 1.50, respectively, and the corresponding percentage of the scale of the local domain subsystems are $15.8 \%$ and $13.6 \%$, respectively. At 5,000th time step, the maximal speedup of METHOD1 and METHOD2 for all the 64 equations are 1.40 and 1.34, respectively, and the corresponding percentage of the scale of the local domains are $43.2 \%$ and $39.8 \%$, respectively.

The comparison of the effectiveness of the methods for some specific linear systems at 1,000th time step is given in Table 3. This table shows that with the 

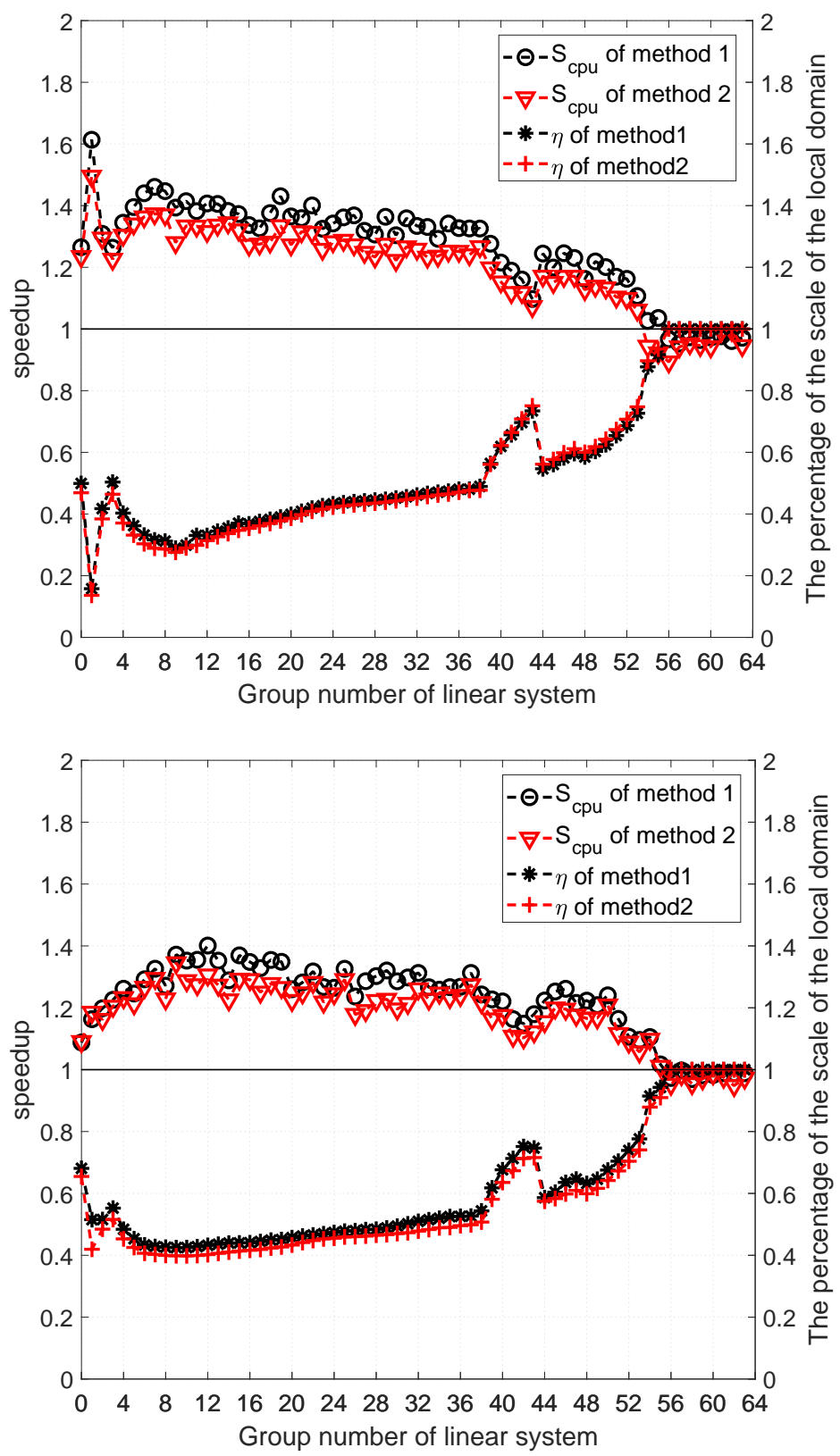

Figure 7: The percentage of the scale of the subsystem and the speedup of the local characterbased methods for solving multi-group radiation diffusion equations. The upper is the result of T1000N1S0G0-G63 at time step 1,000, the lower is the result of T5000N0S0G0-G63 at time step 5,000 . 
Table 3: Speedup of METHOD1 and METHOD2 to METHOD0 for some groups of T1000N1S0.

\begin{tabular}{ccccccc}
\hline G\# & Method & $\eta$ & $S_{\text {CPU }}$ & CPU $_{\text {total }}$ & CPU $_{\text {loc-constr }}$ & CPU loc-solv \\
\hline \multirow{3}{*}{ G1 } & METHOD0 & - & 1.0 & $1.95 \mathrm{E}-2$ & - & - \\
& METHOD1 & $15.8 \%$ & 1.61 & $1.21 \mathrm{E}-2$ & $4.73 \mathrm{E}-3$ & $6.65 \mathrm{E}-3$ \\
& METHOD2 & $13.6 \%$ & 1.50 & $1.30 \mathrm{E}-2$ & $5.62 \mathrm{E}-3$ & $6.74 \mathrm{E}-3$ \\
\hline \multirow{3}{*}{ G10 } & METHOD0 & - & 1.0 & $2.16 \mathrm{E}-2$ & - & - \\
& METHOD1 & $29.9 \%$ & 1.41 & $1.53 \mathrm{E}-2$ & $5.01 \mathrm{E}-3$ & $9.64 \mathrm{E}-3$ \\
& METHOD2 & $29.0 \%$ & 1.33 & $1.62 \mathrm{E}-2$ & $5.68 \mathrm{E}-3$ & $9.89 \mathrm{E}-3$ \\
\hline \multirow{3}{*}{ G11 } & METHOD0 & - & 1.0 & $2.65 \mathrm{E}-2$ & - & - \\
& METHOD1 & $65.7 \%$ & 1.19 & $2.23 \mathrm{E}-2$ & $5.61 \mathrm{E}-3$ & $1.61 \mathrm{E}-2$ \\
& METHOD2 & $66.4 \%$ & 1.12 & $2.37 \mathrm{E}-2$ & $6.40 \mathrm{E}-3$ & $1.67 \mathrm{E}-2$ \\
\hline \multirow{3}{*}{ G55 } & METHOD0 & - & 1.0 & $3.25 \mathrm{E}-2$ & - & - \\
& METHOD1 & $91.7 \%$ & 1.04 & $3.14 \mathrm{E}-2$ & $6.18 \mathrm{E}-3$ & $2.45 \mathrm{E}-2$ \\
& METHOD2 & $93.5 \%$ & 0.92 & $3.52 \mathrm{E}-2$ & $6.74 \mathrm{E}-3$ & $2.78 \mathrm{E}-2$ \\
\hline \multirow{3}{*}{ G63 } & METHOD0 & - & 1.0 & $3.68 \mathrm{E}-2$ & - & - \\
& METHOD1 & $100 \%$ & 0.97 & $3.79 \mathrm{E}-2$ & $6.24 \mathrm{E}-3$ & $3.11 \mathrm{E}-2$ \\
& METHOD2 & $100 \%$ & 0.95 & $3.89 \mathrm{E}-2$ & $6.79 \mathrm{E}-3$ & $3.15 \mathrm{E}-2$ \\
\hline
\end{tabular}

increase of the group number, the speedup of the local character-based method decreases because $\eta$ increases. In particular, when $\eta$ approximates 1 , the time costs of both the local character-based methods are larger than that of METHOD0. Further analysis of the choice of the parameters will be presented in section 3.5 .

In multi-group radiation energy model, the high energy groups represent the energy of high frequency photons. Usually, the number of high frequency photons is small. This makes the energy of high frequency photons low. Particularly, for multi-group radiation diffusion equations, the solution of high energy groups is small in the computing domain, and the local character is not strong. This makes the method ineffective for high energy groups. At the same time, with time advancing, the temperature (representing energy) increases in the simulation domain for multi-group radiation diffusion equations (also for three temperature energy equations), the local character becomes weak. Therefore, the performance of the local character-based method deteriorates at large time steps.

\subsection{Test of three temperature energy equations}

When only one group is used in the multi-group radiation diffusion equations, the multi-group radiation diffusion equations coupled to electron-ion equations are reduced to the three temperature (3T) energy equations, which can be given as follows:

$$
\left\{\begin{array}{l}
C_{v e} \frac{\partial T_{e}}{\partial t}-\frac{1}{\rho} \nabla \cdot\left(K_{e} \nabla T_{e}\right)=\omega_{e i}\left(T_{i}-T_{e}\right)+\omega_{e r}\left(T_{r}-T_{e}\right) \\
C_{v i} \frac{\partial T_{i}}{\partial t}-\frac{1}{\rho} \nabla \cdot\left(K_{i} \nabla T_{i}\right)=\omega_{e i}\left(T_{e}-T_{i}\right) \\
C_{v r} \frac{\partial T_{r}}{\partial t}-\frac{1}{\rho} \nabla \cdot\left(K_{r} \nabla T_{r}\right)=\omega_{e r}\left(T_{e}-T_{r}\right)
\end{array}\right.
$$


In the equations,

- $T_{e}, T_{i}$, and $T_{r}$ are electron temperature, ion temperature, and photon temperature, respectively.

- $C_{v e}, C_{v i}$, and $C_{v r}$ are electron heat capacity, ion heat capacity, and photon heat capacity, respectively.

- $\rho$ is the material density.

- $\omega_{e i}$ and $\omega_{e r}$ are the energy exchanging coefficients between electrons and ions, and that between electrons and photons, respectively.

- $K_{e}, K_{i}$, and $K_{r}$ are electron heat conduction coefficients, ion heat conduction coefficients, and photon heat conduction coefficients, respectively.

Similar discretization methods as for multi-group radiation diffusion equations are used. More details of the 3T equations are presented in [1, 15].

\subsubsection{Linear systems for test}

The test set in this case consists of eight linear systems, which are extracted from the simulation every 10,000 time steps, from the 20,000th time step to the 90,000th time step. After being discretized, the variables of the $3 \mathrm{~T}$ energy equations are organized as follows in the linear system

$$
T=\left(T_{1}^{e}, T_{1}^{i}, T_{1}^{r}, \ldots, T_{M}^{e}, T_{M}^{i}, T_{M}^{r}\right)^{T},
$$

where $M$ is the number of the cells. In this case, $M=9,396$, and the total number of the unknowns in each linear system is 28,188 . For convenience, we denote the linear systems in the test set as $t_{n}$, which represents the linear system at the $n$-th time step. All the coefficient matrices of the linear systems have the same sparsity pattern as is shown in Fig. 8 .

\subsubsection{Test result}

In this test, all the parameters are the same as for the test of the multigroup radiation diffusion equations. The speedups and the percentages of the scale of the local domain are shown in Fig. 9. From this figure, one can see that, for most of the tested linear systems, METHOD1 and METHOD2 are more efficient than METHOD0. The maximal speedup of METHOD1 to METHOD0 is 1.61, and the corresponding percentage of the scale of the local domain is about $30.4 \%$. The maximal speedup of METHOD2 to METHOD0 is about 1.65, and the corresponding percentage of the scale of the local domain is about $25.3 \%$.

The detailed results for four linear systems of $3 \mathrm{~T}$ energy equations are given in Table 4 . From this table, one can see that for the linear equations at time step 20,000, 40,000 and 60,000, the speedups of METHOD1 and METHOD2 are larger than 1.0. For the linear system at time step 80,000 , the speedup is less than one for both METHOD1 and METHOD2. That is, the solution time of METHOD1 and METHOD2 is longer than METHOD0. The parameters in the methods have some influence on the performance, and this will be studied further in the next subsection. 


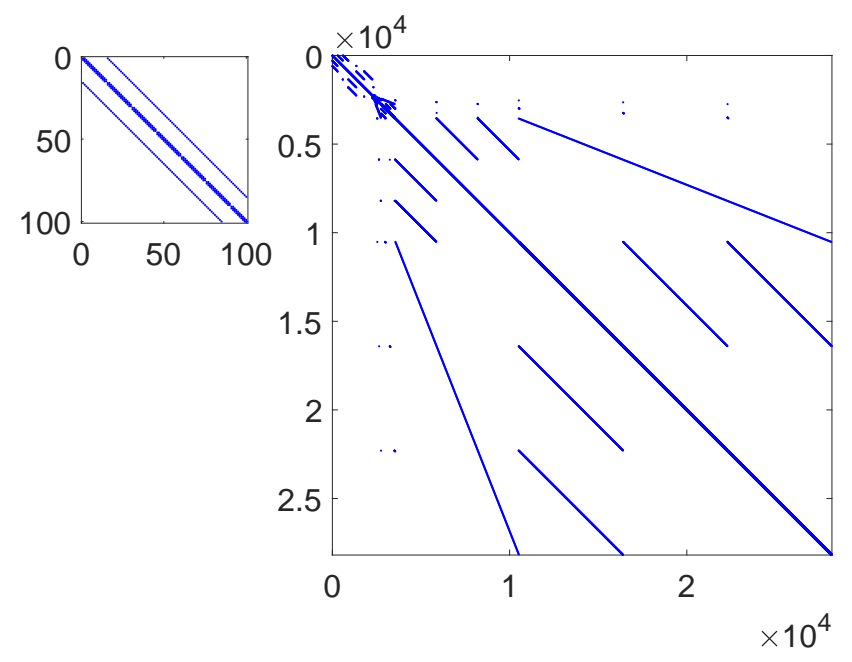

Figure 8: The sparsity pattern of the matrix in linear system of 3T equations.

Table 4: Speedup of METHOD1 and METHOD2 to METHOD0 for 3T energy equations on four time steps.

\begin{tabular}{ccccccc}
\hline$t_{\#}$ & Method & $\eta$ & $S_{\text {CPU }}$ & CPU $_{\text {total }}$ & CPU loc-constr & CPU $_{\text {loc-solv }}$ \\
\hline \multirow{2}{*}{$t_{20000}$} & METHOD0 & - & 1.0 & $4.25 \mathrm{E}-2$ & - & - \\
& METHOD1 & $30.4 \%$ & 1.61 & $2.64 \mathrm{E}-2$ & $9.61 \mathrm{E}-3$ & $1.45 \mathrm{E}-2$ \\
& METHOD2 & $25.3 \%$ & 1.66 & $2.56 \mathrm{E}-2$ & $9.88 \mathrm{E}-3$ & $1.35 \mathrm{E}-2$ \\
\hline \multirow{3}{*}{$t_{40000}$} & METHOD0 & - & 1.0 & $4.85 \mathrm{E}-2$ & - & - \\
& METHOD1 & $43.8 \%$ & 1.44 & $3.38 \mathrm{E}-2$ & $1.10 \mathrm{E}-2$ & $2.05 \mathrm{E}-2$ \\
& METHOD2 & $40.2 \%$ & 1.48 & $3.28 \mathrm{E}-2$ & $1.11 \mathrm{E}-2$ & $1.94 \mathrm{E}-2$ \\
\hline \multirow{3}{*}{$t_{60000}$} & METHOD0 & - & 1.0 & $4.85 \mathrm{E}-2$ & - & - \\
& METHOD1 & $61.1 \%$ & 1.21 & $3.99 \mathrm{E}-2$ & $1.17 \mathrm{E}-2$ & $2.59 \mathrm{E}-2$ \\
& METHOD2 & $58.1 \%$ & 1.19 & $4.06 \mathrm{E}-2$ & $1.25 \mathrm{E}-2$ & $2.57 \mathrm{E}-2$ \\
\hline \multirow{3}{*}{$t_{80000}$} & METHOD0 & - & 1.0 & $5.77 \mathrm{E}-2$ & - & - \\
& METHOD1 & $88.8 \%$ & 0.95 & $6.10 \mathrm{E}-2$ & $1.50 \mathrm{E}-2$ & $4.34 \mathrm{E}-2$ \\
& METHOD2 & $87.8 \%$ & 0.68 & $1.01 \mathrm{E}-1$ & $1.56 \mathrm{E}-2$ & $4.10 \mathrm{E}-2$ \\
\hline
\end{tabular}




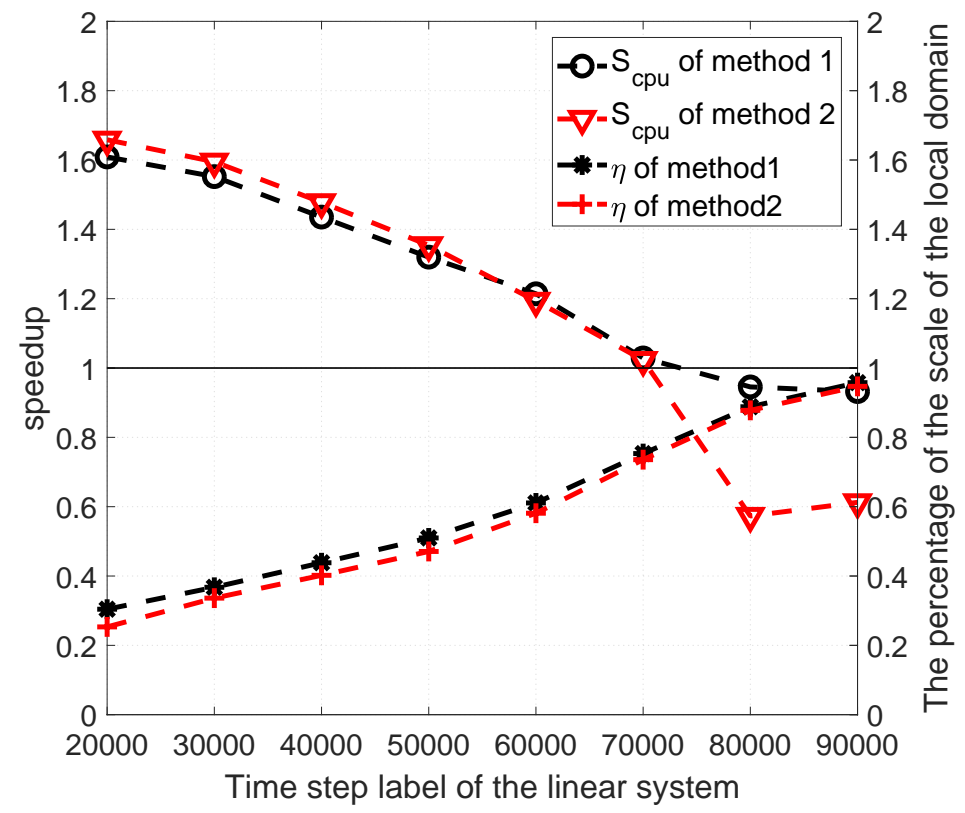

Figure 9: The percentage of the scale of the subsystem and the speedup of the local characterbased methods for solving $3 \mathrm{~T}$ energy equations.

\subsection{Influences of the parameters}

In both Algorithm 2 and Algorithm 3, the parameters should be set for specific applications. In the previous tests, the parameters are fixed. In this subsection, the influence of the parameters for the algorithms will be analyzed.

\subsubsection{The parameter $\alpha$ in METHOD1}

In METHOD1, $\alpha$ is used to construct the local domain $\Omega_{\text {local }}$. Therefore, $\alpha$ has a significant influence on the effectiveness of the method. When $\alpha$ is too small, the scale of the subsystem (measured by $\eta$, the percentage of the scale of the local domain subsystem to the scale of the global system) will be large. This will deteriorate the performance of METHOD1. Conversely, when $\alpha$ is too large, $\eta$ will be small, and the local domain $\Omega_{\text {local }}$ may not be large enough to capture the physical information being propagated forward in time. This will further decrease the performance of the local character-based method. In particular, if $\alpha=0$, then $\Omega_{\text {local }}=\Omega$, the whole domain; if $\alpha=1$, then $\Omega_{\text {local }}=\emptyset$. For a linear system, one way to determine the optimal value of $\alpha$ is

$$
\alpha_{\mathrm{opt}}=\arg \min _{\alpha}\left\{\alpha:\left\|b-A \tilde{x}_{\alpha}\right\|_{2} \leq \epsilon\|b\|_{2}\right\},
$$

where $\tilde{x}_{\alpha}$ is the solution obtained by the local character-based method, and the local domain is constructed with the the parameter $\alpha$. 
To show the influence of the parameter $\alpha$ on the method, two linear systems of multi-group radiation diffusion equations, T1000N1S0G0 and T1000N1S0G55, are selected. The value of $\alpha$ is set to 12 different orders of magnitude values, varying from 1 to $10^{-11}$.

Fig. 10 shows the speedups of the local character-based method and the percentage of the scale of the local domain for both cases. The results show that $\eta=\eta(\alpha)$ is monotonic decreasing, which is consistent with the construction of the local character-based method. For the tested two linear systems, the $\alpha$ values corresponding to the maximal speedup are $10^{-7}$ and $10^{-10}$, respectively, and the maximal speedups are 1.38 and 1.1, respectively. Particularly, for the linear system T1000N1S0G55, this figure shows that the speedups are lower than 1 for most of the cases. This indicates that METHOD1 is not a good candidate for solving this linear equation because the local character of this linear system is not so strong.

\subsubsection{The parameter $E_{\max }$ in METHOD2}

In METHOD2, $E_{\max }$ is used in the second stage of the local domain construction method, and it represents the maximal expanding iterations for the local domain $\Omega_{\text {local }}$. Theoretically, a large enough value for $E_{\max }$ can be set so that $\Omega_{\text {local }}$ cannot be expanded anymore. However, the larger that $E_{\max }$ is, the more expensive constructing the local character-based method will be. An improper $E_{\max }$ may result in a loss of speedup of the method. To show the specific influence of the parameter $E_{\max }$, two linear systems of $3 \mathrm{~T}$ energy equations, $t_{20000}$ and $t_{80000}$, are tested further with different $E_{\max }$. In the test, the value of $E_{\max }$ varies from 0 to 6 . The test results are shown in Fig. 11.

From Fig. 11, one can see that, for linear system $t_{20000}, \eta$ does not have clear increase when $E_{\text {max }}$ increases from 1 to 6 , and the maximal speedup is 1.66 when $E_{\max }=1$. This means that $E_{\max }=1$ is the optimal value for solving this linear system. For linear system $t_{80000}$, again the value of $\eta$ does not increase clearly when $E_{\max }$ increases from 1 to 6 . However, the speedup has a dramatic increase when $E_{\max }$ increases from 3 to 4 , and the optimal $E_{\max }$ value is 4 for solving this linear system. However, for this linear system, the speedups are less than 1 for all cases. This means that the local character-based method is not good at solving the linear system $t_{80000}$ because its local character is not so strong.

\section{Summary and remarks}

The solution of radiation diffusion equations is very important in the simulation of inertial confinement fusion (ICF) and astrophysics. The solution of radiation diffusion equations concerns many linear equation sequences with the total number of linear equations is about $\mathcal{O}\left(10^{4}\right)$. The radiation diffusion equations are time-dependent nonlinear systems and the solution at the previous time step or nonlinear iterate is usually used as the initial iterate for solving the linear equations. Furthermore, in most of the simulation period, the solution only varies greatly in some local domain. 

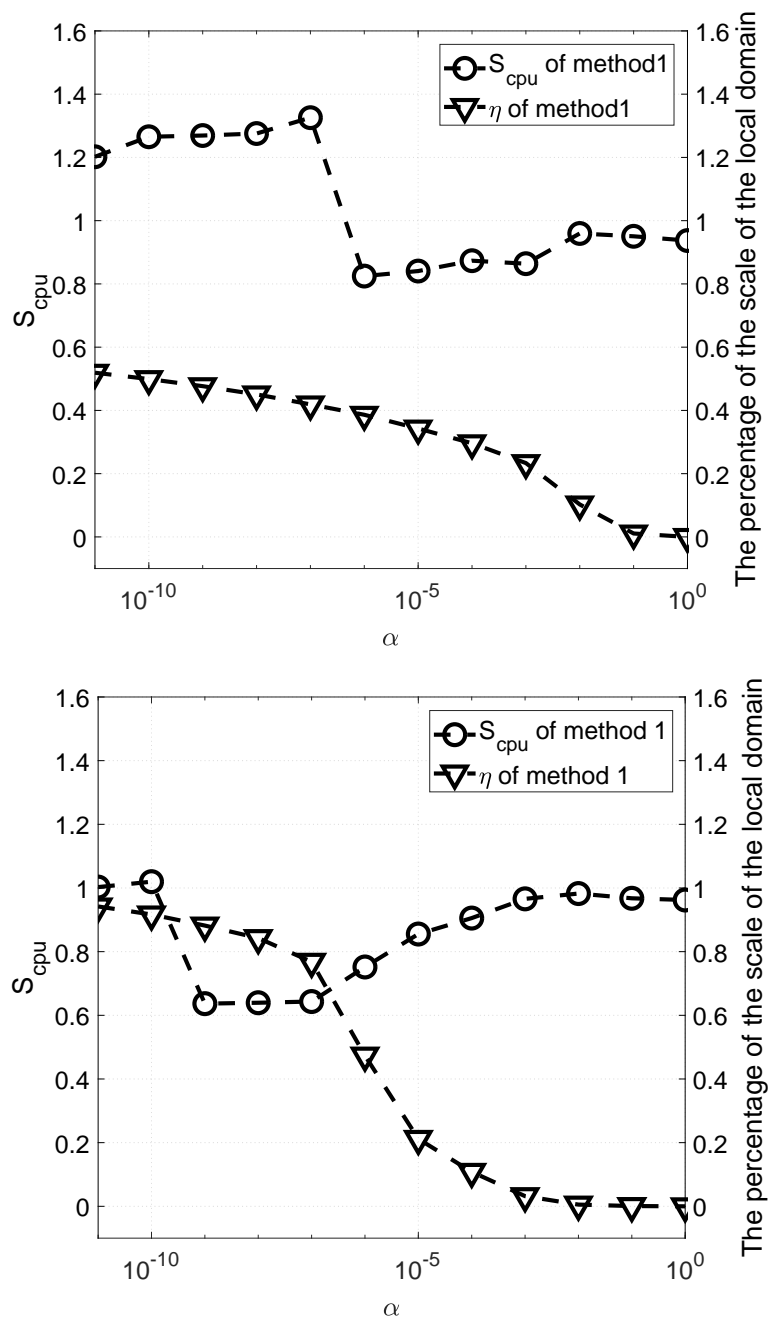

Figure 10: The percentage of scale of the local domain and speedup of METHOD1 with different $\alpha$. The upper sub-figure is the result of T1000N1S0G0, the lower sub-figure is the result of T1000N1S0G55. 

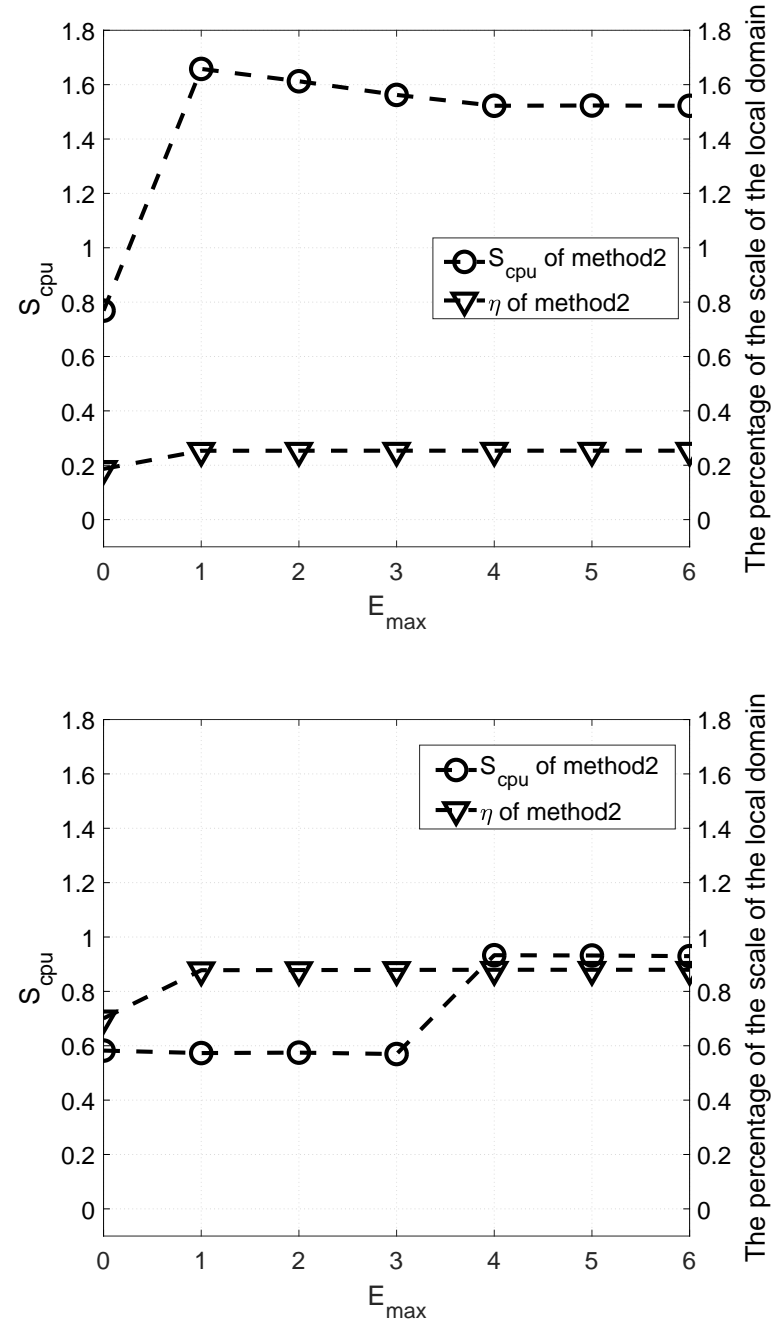

Figure 11: The percentage of scale of the local domain and speedup of METHOD2 with different $E_{\max }$. The upper sub-figure is the result of $t_{20000}$, the lower sub-figure is the result of $t_{80000}$. 
In this paper, a local character-based method is proposed to solve the linear systems in this application. In the local character-based method, first, a local domain is constructed. It is expected that on the local domain the solution varies greatly from the initial iterate to the last iterate solution. Then the subsystem on the local domain is solved. At last, the global linear system is solved with the initial iterate obtained by solving the local domain system. Two methods are proposed to construct the local domain. One is based on the spatial gradient, and the other is based on the initial residual. Therefore, two kinds of specific methods are constructed.

Numerical results show that the proposed local character-based methods can successfully grasp the local characteristic domain. A two-dimensional heat conduction equation is tested and analyzed. Also, the linear systems from real applications of multi-group radiation diffusion equations and 3T energy equations are tested. Compared to solving the linear system directly by some method, such as preconditioned Krylov methods, the solution time can be reduced by $38 \%$ and $40 \%$ respectively by using the two local character-based methods.

The performance of the local character-based method depends strongly on the percentage of the scale of the local domain subsystem to the scale of the global system. Numerical results for multi-group radiation diffusion equations and $3 \mathrm{~T}$ energy equations show that if the percentage is relatively small, then the local character-based method may perform better than the typical method, such as AMG preconditioned GMRES method. Otherwise, the local characterbased method may perform worse than the typical method. Therefore, in the simulation of real applications, if the temperature is not high in the whole simulation domain, then the local character is strong, and the method proposed in this paper will be efficient. In the later simulation period, with the increase of the temperature in the whole domain, the local character may not be so strong, the typical method should be used. In the future, we will consider constructing the preconditioning method based on the local character-based method.

\section{References}

[1] H. An, Z. Mo, X. Xu, X. Liu, On choosing a nonlinear initial iterate for solving the 2-D 3-T heat conduction equations, Journal of Computational Physics 228(9) (2009) 3268-3287.

[2] C. Baldwin, P.N. Brown, R. Falgout, F. Graziani, J. Jones, Iterative linear solvers in a 2D radiation-hydrodynamics code: Methods and performance, Journal of Computational Physics 154(1) (1999) 1-40.

[3] M.F. Barone, I. Kalashnikova, D.J. Segalman, H.K. Thornquist, Stable galerkin reduced order models for linearized compressible flow, Journal of Computational Physics 228(6) (2009) 1932-1946.

[4] T.F. Chan, M.K. Ng, Galerkin projection methods for solving multiple linear systems, SIAM Journal on Scientific Computing 21(3) (1999) 836850. 
[5] T.F. Chan, W.L. Wan, Analysis of projection methods for solving linear systems with multiple right-hand sides, SIAM Journal on Scientific Computing 18(6) (1997) 1698-1721.

[6] R. Djeddi, A. Kaminsky, K. Ekici, Convergence acceleration of fluid dynamics solvers using a reduced-order model, AIAA Journal 55(9) (2017) 3059-3071.

[7] P.F. Fischer, Projection techniques for iterative solution of $A x=b$ with successive right-hand sides, Computer methods in applied mechanics and engineering 163(1-4) (1998) 193-204.

[8] S. Gong, X. Cai, A nonlinear elimination preconditioned Newton method with applications in arterial wall simulation, International Conference on Domain Decomposition Methods, pages 353-361. Springer, 2017.

[9] L. Grinberg, G.E. Karniadakis, Extrapolation-based acceleration of iterative solvers: Application to simulation of 3D flows, Communications in Computational Physics 9(3) (2011) 607-626.

[10] K.C. Hall, J.P. Thomas, E.H. Dowell, Proper orthogonal decomposition technique for transonic unsteady aerodynamic flows, AIAA journal 38(10) (2000) 1853-1862.

[11] X. Hang, J. Li, G. Yuan, Convergence analysis on splitting iterative solution of multi-group radiation diffusion equations, Chinese Journal of Computational Physics 30(1) (2013) 111-119.

[12] J. Huang, C. Yang, X. Cai, A nonlinearly preconditioned inexact Newton algorithm for steady state lattice Boltzmann equations, SIAM Journal on Scientific Computing 38(3) (2016) A1701-A1724.

[13] D.A. Knoll, W.J. Rider, G.L. Olson, Nonlinear convergence, accuracy, and time step control in nonequilibrium radiation diffusion, Journal of Quantitative Spectroscopy and Radiative Transfer 70(1) (2001) 25-36.

[14] R. Markovinović, J.D. Jansen, Accelerating iterative solution methods using reduced-order models as solution predictors, International journal for numerical methods in engineering 68(5) (2006) 525-541.

[15] Z. Mo, L. Shen, G. Wittum, Parallel adaptive multigrid algorithm for 2D 3-T diffusion equation, International Journal of Computer Mathematics 81(3) (2004) 361-374.

[16] J.E. Morel, E.W. Larsen, M.K. Matzen, A synthetic acceleration scheme for radiative diffusion calculations, Journal of Quantitative Spectroscopy and Radiative Transfer 34(3) (1985) 243-261.

[17] V.A. Mousseau, D.A. Knoll, W.J. Rider, Physics-based preconditioning and the Newton-Krylov method for non-equilibrium radiation diffusion, Journal of computational physics 160(2) (2000) 743-765. 
[18] J.W. Ruge, K. Stüben, Algebraic multigrid, pages 73-130. SIAM, 1987.

[19] Y. Saad, Iterative methods for sparse linear systems, SIAM, 2003.

[20] K.S. Shterev, Iterative process acceleration of calculation of unsteady, viscous, compressible, and heat-conductive gas flows, International Journal for Numerical Methods in Fluids 77(2) (2015) 108-122.

[21] P. Song, C. Zhai, S. Li, Lared-integration code for numerical simulation of the whole process of the indirect-drive laser inertial confinement fusion, High Power Laser and Particle Beams 27(3), 2015.

[22] D. Tromeur-Dervout, Y. Vassilevski. Choice of initial guess in iterative solution of series of systems arising in fluid flow simulations, Journal of Computational Physics 219(1) (2016) 210-227.

[23] X. Xu, Z. Mo, H. An. An adaptive AMG preconditioning strategy for solving large-scale sparse linear systems, Scientia Sinica Infromationis 46(10) (2016) 1411-1420.

[24] H. Yang, F.N. Hwang, X. Cai, Nonlinear preconditioning techniques for fullspace Lagrange-Newton solution of PDE-constrained optimization problems, SIAM Journal on Scientific Computing 38(5) (2016) A2756-A2778.

[25] G. Yuan, X. Hang, Z. Sheng, J. Yue, Progress in numerical methods for radiation diffusion equations, Chinese Journal of Computational Physics 26(4) (2009) 475-500. 